\title{
The Theory of Higher-Order Types of Asymptotic Variation for Differentiable Functions. Part I: Higher-Order Regular, Smooth and Rapid Variation
}

\author{
Antonio Granata \\ Department of Mathematics and Computer Science, University of Calabria, Rende (Cosenza), Italy \\ Email: antoniogranata1973@gmail.com
}

How to cite this paper: Granata, A. (2016) The Theory of Higher-Order Types of Asymptotic Variation for Differentiable Functions. Part I: Higher-Order Regular, Smooth and Rapid Variation. Advances in Pure Mathematics, 6, 776-816.

http://dx.doi.org/10.4236/apm.2016.612063

Received: September 7, 2016

Accepted: November 7, 2016

Published: November 10, 2016

Copyright $\odot 2016$ by author and Scientific Research Publishing Inc. This work is licensed under the Creative Commons Attribution International License (CC BY 4.0).

http://creativecommons.org/licenses/by/4.0/

\section{(c) () Open Access}

\begin{abstract}
Motivated by a general theory of finite asymptotic expansions in the real domain for functions $f$ of one real variable, a theory developed in a previous series of papers, we present a detailed survey on the classes of higher-order asymptotically-varying functions where "asymptotically" stands for one of the adverbs "regularly, smoothly, rapidly, exponentially". For order 1 the theory of regularly-varying functions (with a minimum of regularity such as measurability) is well established and well developed whereas for higher orders involving differentiable functions we encounter different approaches in the literature not linked together, and the cases of rapid or exponential variation, even of order 1, are not systrematically treated. In this semi-expository paper we systematize much scattered matter concerning the pertinent theory of such classes of functions hopefully being of help to those who need these results for various applications. The present Part I contains the higher-order theory for regular, smooth and rapid variation.
\end{abstract}

\section{Keywords}

Higher-Order Regularly-Varying Functions, Higher-Order Rapidly-Varying Functions, Smoothly-Varying Functions, Exponentially-Varying Functions, Asymptotic Functional Equations

\section{Introduction}

In a previously-published series of papers ([1] [2] [3] [4] [5]) we established a general analytic theory of finite asymptotic expansions in the real domain for functions $f$ of 
one real variable sufficiently regular on a deleted neighborhood of a point $x_{0} \in \overline{\mathbb{R}}$. The theory is based on the use of a uniquely-determined linear differential operator $L_{\phi_{1}, \cdots, \phi_{n}}$ associated to a given asymptotic scale " $\phi_{1}(x) \gg \phi_{2}(x) \gg \cdots \gg \phi_{n}(x), x \rightarrow x_{0}^{-}$”; and the conditions characterizing two sets of expansions obtained from

$$
f(x)=a_{1} \phi_{1}(x)+\ldots+a_{n} \phi_{n}(x)+o\left(\phi_{n}(x)\right), x \rightarrow x_{0}^{-},
$$

by two special procedures of formal differentiation are expressed via improper integrals involving both the quantity $L_{\phi_{1}, \cdots, \phi_{n}}[f(x)]$ and certain ratios of Wronskians of the $\phi_{i}$ 's. For instance one such condition is

$$
\int_{T}^{\rightarrow x_{0}} \frac{W\left(\phi_{1}(t), \cdots, \phi_{n-1}(t)\right)}{W\left(\phi_{1}(t), \cdots, \phi_{n}(t)\right)} L_{\phi_{1}, \cdots, \phi_{n}}[f(t)] \mathrm{d} t \text { convergent, }
$$

and for practical applications it is quite useful to have some information on the asymptotic behavior of the ratio of Wronskians. For $x_{0}=+\infty$ a possible step consists in writing

$$
\left|\begin{array}{ccc}
\phi_{1}(x) & \cdots & \phi_{n}(x) \\
\phi_{1}^{\prime}(x) & \cdots & \phi_{n}^{\prime}(x) \\
\vdots & & \vdots \\
\phi_{1}^{(n-1)}(x) & \cdots & \phi_{n}^{(n-1)}(x)
\end{array}\right|=\left(\prod_{i=1}^{n} \phi_{i}(x)\right) \cdot\left|\begin{array}{ccc}
1 & \cdots & 1 \\
\phi_{1}^{\prime}(x) / \phi_{1}(x) & \cdots & \phi_{n}^{\prime}(x) / \phi_{n}(x) \\
\phi_{1}^{\prime \prime}(x) / \phi_{1}(x) & \cdots & \phi_{n}^{\prime \prime}(x) / \phi_{n}(x) \\
\vdots & & \vdots \\
\phi_{1}^{(n-1)}(x) / \phi_{1}(x) & \cdots & \phi_{n}^{(n-1)}(x) / \phi_{n}(x)
\end{array}\right|,
$$

and then recalling that in various but related contexts the following remarkable asymptotic relations linking the ratios $\phi^{(k)}(x) / \phi(x)$ to $\phi^{\prime}(x) / \phi(x)$ are found:

$$
\frac{\phi^{(k)}(x)}{\phi(x)}=\left(\frac{\phi^{\prime}(x)}{\phi(x)}\right)^{k}\left[\frac{(\alpha-1) \cdots(\alpha-k+1)}{\alpha^{k-1}}+o(1)\right], x \rightarrow+\infty, 1 \leq k \leq n \text {; }
$$

or

$$
\frac{\phi^{(k)}(x)}{\phi(x)} \sim\left(\frac{\phi^{\prime}(x)}{\phi(x)}\right)^{k}, x \rightarrow+\infty, 1 \leq k \leq n ;
$$

for different classes of functions. Bourbaki ([6]; Chap. V, appendix, pp. V.36-V.40), in the context of Hardy fields, shows their validity for each $k \in \mathbb{N}$ (some exceptional cases apart) for the classes of functions therein called "of finite order $\alpha$ [respectively, of infinite order] with respect to the function $g(x):=x$, as $x \rightarrow+\infty$ " and defined by the property that

$$
\lim _{x \rightarrow+\infty} \log |\phi(x)| / \log x \text { exists in } \overline{\mathbb{R}} \text {. }
$$

Balkema, Geluk and de Haan, ([7]; Lemma 9, p. 410), have shown the equivalence of (1.4), written in the form

$$
\frac{\phi^{(k)}(x)}{\phi(x)}=\alpha(\alpha-1) \cdots(\alpha-k+1) x^{-k}+o\left(x^{-k}\right), x \rightarrow+\infty, 1 \leq k \leq n,
$$

with the property that the function $h(x):=\log \left(\phi\left(\mathrm{e}^{x}\right)\right)$ satisfies 


$$
\lim _{x \rightarrow+\infty} h^{(k)}(x)= \begin{cases}\alpha & \text { if } k=1, \\ 0 & \text { if } 2 \leq k \leq n .\end{cases}
$$

$C^{\infty}$-functions satisfying (1.7)-(1.8) for $n \in \mathbb{N}$ are called "smoothly varying at $+\infty$ with exponent (or index) $\alpha$ ": see also Bingham, Goldie and Teugels ([8]; p. 44). Another author, Lantsman ([9]: pp. 96-98), shows the validity of (1.7) for the class of functions such that

$$
\frac{\mathrm{d}^{k}}{\mathrm{~d} x^{k}}\left(\frac{x \phi^{\prime}(x)}{\phi(x)}\right)=\left\{\begin{array}{ll}
\alpha+o(1) & \text { if } k=0 \\
o\left(x^{-k}\right) & \text { if } k \geq 1 ;
\end{array}, x \rightarrow+\infty\right.
$$

such a $\phi$ being said to have a "power order of growth $\alpha$ at $+\infty$ ".

All these approaches for infinitely-differentiable functions have in common the existence of the following limit

$$
\lim _{x \rightarrow+\infty} x f^{\prime}(x) / f(x)= \begin{cases}0 & \text { (slow variation at }+\infty), \\ \alpha \in \mathbb{R} \backslash\{0\} & (\text { regular variation at }+\infty), \\ \pm \infty & (\text { rapid variation at }+\infty),\end{cases}
$$

wherein the three contingencies are special cases of the more general classes of functions traditionally labelled as "slowly, regularly or rapidly varying at $+\infty$ ". Motivated by the fact that the $\phi_{i}^{(k)}$ 's of most asymptotic scales found in applications belong to one of these classes and that the before-mentioned relations for the derivatives have important applications in several fields, we deemed it convenient to systematize the theory of higher-order "types of asymptotic variation" showing the equivalence of various approaches, putting together a large amount of basic properties and highlighting the parallel theory of rapid or exponential variation always cursorily treated. Many proofs have an elementary character left apart: the equivalence of the various approaches based on a remarkable device by Balkema, Geluk and de Haan, and the operations on higher-order varying functions which requires a certain amount of patience. Much time has been spent in giving an abundance of counterexamples to show the necessity of possible restrictive assumptions. A special attention has been paid to listing a variety of asymptotic functional equations satisfied by the functions in the studied classes. Only the general theory has been treated in this semi-expository paper and the applications are restricted to some asymptotic properties of antiderivatives and sums, and to asymptotic expansions of an expression of type $f(x+r(x))$. Applications to general asymptotic expansions and differential-functional equations would require a separate treatment. The exposition is on a plain level and an effort has been made to look for the simplest proofs.

- $\$ 2$ contains a detailed and integrated exposition of basic properties (algebraic, differential and asymptotic) concerning regular and rapid variation in the strong sense. Much, but not all, the material concerning regular variation is standard and the most elementary proofs have been reported. Some facts concerning the index of variation of the first derivative in $\$ 2.3$ are essential both to give a correct definition of higher-order regular variation and to understand possible restrictions on the indexes. 
- In $\$ 3$ we give an integrated exposition of higher-order regular variation (a concept indirectly encountered in the context, e.g., of Hardy fields) and smooth variation (a concept explicitly present in the literature concerning some applications of regular variation), both traditionally (but not in our approach) referred to $C^{\infty}$-functions. We show the equivalence of different approaches found in the literature reporting a clarified version of a non-trivial characterization by Balkema, Geluk and de Haan trying to highlight the computational ideas in the ingenious proof, somehow hidden in the original exposition.

- In $\$ 4$ an analogous exposition for higher-order rapid variation is given with several characterizations. To be useful for applications a restriction must be added to the “spontaneous" concept of higher order for this class of functions.

- In $\$ 5$ there is a discussion about various useful asymptotic functional equations satisfied by the functions in the previously-studied classes.

In part II we exhaustively describe results about algebraic operations on higher-order asymptotically-varying functions and treat concepts related to exponential variation and some of their basic applications.

\section{General notations}

- $\mathbb{N}:=\{1,2, \cdots\} ; \mathbb{Z}:=\{0, \pm 1, \pm 2, \cdots\} ;$

- $\mathbb{R}:=$ real line; $\overline{\mathbb{R}} \equiv$ extended real line $:=\mathbb{R} \cup\{ \pm \infty\}$;

- $f \in A C^{0}(I) \equiv A C(I) \Leftrightarrow f$ is absolutely continuous on each compact subinterval of the interval $I$;

- $f \in A C^{k}(I) \Leftrightarrow f^{(k)} \in A C(I)$;

- For $f \in A C^{k}(I)$ we write " $\lim _{x \rightarrow x_{0}} f^{(k+1)}(x)$ " meaning that $x$ runs through the points wherein $f^{(k+1)}$ exists as a finite number; $f(+\infty):=\lim _{x \rightarrow+\infty} f(x)$.

- If $f \geq 0$ the usual notation $\int^{\infty} f<+\infty \quad[$ resp. $=+\infty]$ means that the improper integral $\int_{T}^{+\infty} f$ is either convergent or divergent for some Tlarge enough.

- The logarithmic derivative $D_{\ell} f:=f^{\prime} / f \equiv(\log |f|)^{\prime}$.

- Hardy's notations:

“ $f(x) \ll g(x), x \rightarrow x_{0}$," or, equivalently “ $g(x) \gg f(x), x \rightarrow x_{0}$," stands for $f(x)=o(g(x)), x \rightarrow x_{0}$;

“ $f(x) \preccurlyeq g(x), x \rightarrow x_{0}$, " or, equivalently “ $g(x) \succcurlyeq f(x), x \rightarrow x_{0}$," stands for $f(x)=O(g(x)), x \rightarrow x_{0}$.

- The relation " $f(x) \asymp g(x), x \rightarrow x_{0}$," which we label as "asymptotic similarity", means that

$c_{1}|g(x)| \leq|f(x)| \leq c_{2}|g(x)| \forall x$ in a deleted neighborhood of $x_{0}\left(c_{i}=\right.$ constant $\left.>0\right)$.

- The relation of asymptotic equivalence:

$$
f(x) \sim g(x), x \rightarrow x_{0} \text {, stands for } f(x)=g(x)[1+o(1)], x \rightarrow x_{0} .
$$

- When describing properties related to exponential variation it is convenient to use the following nonstandard notation:

$$
f(x)=+\infty(g(x)), x \rightarrow x_{0}(x \in \mathcal{I}), \stackrel{\text { def }}{\Leftrightarrow}\left\{\begin{array}{l}
f(x)=h(x) g(x) \forall x \text { near } x_{0}, \\
\lim _{x \rightarrow x_{0}, x \in \mathcal{I}} h(x)=+\infty
\end{array}\right.
$$


and a similar definition for notation $f(x)=-\infty(g(x)), x \rightarrow x_{0}(x \in \mathcal{I})$. In particular:

$$
f(x)= \pm \infty(1), x \rightarrow x_{0}(x \in \mathcal{I}), \stackrel{\text { def }}{\Leftrightarrow} \lim _{x \rightarrow x_{0}, x \in \mathcal{I}} f(x)= \pm \infty .
$$

We shall formally use these notations like the familiar " $O(g(x))$ ” writing, e.g.,

$$
\left\{\begin{array}{l}
{\left[+\infty\left(f_{1}(x)\right)\right] \cdot\left[+\infty\left(f_{2}(x)\right)\right]=+\infty\left(f_{1}(x) f_{2}(x)\right) ;} \\
{\left[+\infty\left(f_{1}(x)\right)\right]+\left[+\infty\left(f_{2}(x)\right)\right]=+\infty\left(f_{1}(x)+f_{2}(x)\right) \text { if } f_{1}, f_{2} \geq 0 .}
\end{array}\right.
$$

- Factorial powers:

$$
\alpha^{0}:=1 ; \alpha^{1}:=\alpha ; \alpha^{\underline{k}}:=\alpha(\alpha-1) \cdots(\alpha-k+1) ; \alpha \in \mathbb{C}, k \in \mathbb{N} ;
$$

where $\alpha^{\underline{k}}$ is termed the " $k$-th falling (三decreasing) factorial power of $\alpha$ ". Notice that we have defined $0^{0}:=1$, hence a linear combination such as $\sum_{i=1}^{n} a_{i}\left(\alpha_{i}\right)^{0}$ simply means $\sum_{i=1}^{n} a_{i}$ whatever the $\alpha_{i}$ 's.

Propositions are numbered consecutively in each section irrespective of their labelling as lemma, theorem and so on.

Notations for iterated natural logarithms and exponentials

$$
\begin{gathered}
\ell_{k}(x): \underbrace{\log (\log (\cdots(\log x) \cdots))}_{k}, k \geq 1,(\text { defined for } x \text { large enough }) ; \ell_{0}(x):=x ; \\
\exp _{k}(x):=\underbrace{\exp (\exp (\cdots(\exp x) \cdots))}_{k}, k \geq 1 ; \exp _{0}(x):=1 .
\end{gathered}
$$

The special definitions for $\ell_{0}$ and $\exp _{0}$ are agreements. Their derivatives are:

$$
\frac{\mathrm{d}}{\mathrm{d} x}\left(\ell_{k}(x)\right)=\left(\prod_{i=0}^{k-1} \ell_{i}(x)\right)^{-1}, k \geq 1 ; \quad \frac{\mathrm{d}}{\mathrm{d} x}\left(\exp _{k}(x)\right)=\prod_{i=1}^{k} \exp _{i}(x), k \geq 1 .
$$

\section{The Elementary Concept of "Index of Variation" and Properties of Related Functions}

The general theory of finite asymptotic expansions we constructed in the cited papers essentially deals with functions of the regularity class $C^{n}[T,+\infty)$ or, with mathematical pedantry, of class $A C^{n-1}[T,+\infty)$; for consistency we need asymptotic relations for the ratios $\phi_{i}^{(k)}(x) / \phi_{i}(x)$ appearing in the right-hand side of (1.3) for functions with such regularity with no additional restrictions either of algebraic character or of $C^{\infty}$ -regularity such as in the theory of Hardy fields, Bourbaki ([6]; Chap. V, Appendix), or in other expositions, Lantsman ([9]; Chap. 5). In the modern well-developed-and-organized theory of regular or rapid variation, with its many applications to probability and statistics, the approach via (1.10) is of secondary importance but for higher-order variation the "natural" approach is that of introducing $C^{n}$-functions whose all derivatives have an index of variation just in the sense of (1.10); and it will be seen that an additional condition is required for rapid variation. Both for applications and for further theoretical results we need many of the standard properties of regularly- or rapidly-varying functions and so we cannot help giving an almost complete list of them though their proofs are usually elementary even not always obvious; not all of those in- 
volving rapid variation are to be found in texts on the subject. A special attention is given to linear combinations of asymptotic scales. As concerns higher-order variation the essential fact that the classes of higher-order regularly- or rapidly-varying functions are closed with respect to the operations of product, composition and inversion requires nontrivial proofs reported in Part II. The results will make the reader feel quite at ease with the many examples scattered in our work. The asymptotic relations for the ratios $\phi_{i}^{(k)}(x) / \phi_{i}(x)$ obviously are those familiar in the context of Hardy fields but our context is more general and some useful points about the indexes are highlighted in certain exceptional cases.

Unlike the traditional concept of "order of growth" which involves one specified comparison function we use the generic locution of "type of growth", or better "type of asymptotic variation", to denote one of the classes of functions which are either regularly or smoothly or rapidly or exponentially varying; and these are classes which in our exposition are defined via "asymptotic differential equations" whereas for order 1 they may be included in larger classes defined through "asymptotic functional equations".

\subsection{The Elementary Concept of "Index of Variation"}

Definition 2.1. Let $f \in A C[T,+\infty), f(x)>0$ for each $x$ large enough.

(I) $f$ is termed "regularly varying at $+\infty$ (in the strong sense)" if

$$
f^{\prime}(x) / f(x)=\alpha x^{-1}+o\left(x^{-1}\right), x \rightarrow+\infty,
$$

for some constant $\alpha \in \mathbb{R}$ which is called the index of regular variation of $f$ at $+\infty$. We denote the family of all such functions for a fixed $\alpha$ by $\mathcal{R}_{\alpha}(+\infty)$. In the case $\alpha=0$ the function $f$ is also termed "slowly varying at $+\infty$ (in the strong sense)".

(II) $f$ is termed "rapidly varying at $+\infty$ (in the strong sense)" if

$$
\lim _{x \rightarrow+\infty} x f^{\prime}(x) / f(x)= \pm \infty .
$$

Accordingly, the index of rapid variation at $+\infty$ is defined to be either $+\infty$ or $-\infty$ and the corresponding families of functions are denoted by $\mathcal{R}_{+\infty}(+\infty)$ and $\mathcal{R}_{-\infty}(+\infty)$; we also put $\mathcal{R}_{\infty}(+\infty):=\mathcal{R}_{+\infty}(+\infty) \cup \mathcal{R}_{-\infty}(+\infty)$.

(III) $f$ is said to have an "index of variation at $+\infty$ in the strong sense" if the following limit exists in the extended real line.

$$
\lim _{x \rightarrow+\infty} x f^{\prime}(x) / f(x) \equiv \alpha,-\infty \leq \alpha \leq+\infty,
$$

with the tacit agreement that the limit is taken for $x$ such that $f^{\prime}(x)$ exists as a finite number. Whenever there is no need to specify the index of variation we denote the class of all such functions by the symbol

$$
\mathcal{R}(+\infty):=\bigcup_{\alpha \in \mathbb{R}} \mathcal{R}_{\alpha}(+\infty) \cup \mathcal{R}_{+\infty}(+\infty) \cup \mathcal{R}_{-\infty}(+\infty) .
$$

We sometimes omit the specification "in strong sense" as this is the only meaning we are using for this concept.

Remarks. 1. Condition " $f$ ultimately of one strict sign" is essential both in the general and in our restricted definition. The choice $f>0$ is merely conventional. Writing 
$|f| \in R(+\infty)$ tacitly implies " $f \in A C[T,+\infty)$ for some $T$ and $f(x) \neq 0$ for $x$ large enough". However in some cases the positivity of $f$ may be essential for a correct result as when investigating the possible variation-properties of a linear combination.

2. The locution "in strong sense" is a reminder of the fact that our class of functions is a proper subset of the class of regularly- or rapidly-varying functions in more general senses. The first larger class is that of those real-valued functions $f$ defined on a neighborhood of $+\infty$ and admitting of an "order $\alpha$ ", with respect to the comparison function $g(x):=x$, defined by

$$
\alpha \equiv \lim _{x \rightarrow+\infty} \log |f(x)| / \log x,-\infty \leq \alpha \leq+\infty,
$$

according to Definition 5 in Bourbaki ([6]; p. V.9) where the obviously-misprinted quantity $\log (|f| / \log g)$ stands for $\log |f| / \log g$. By L'Hospital's rule (2.3) trivially implies (2.5). A second still larger class, namely the Karamata class, contains those positive measurable functions $f$ such that

$$
f(\lambda x) \sim \lambda^{\alpha} f(x), x \rightarrow+\infty \text {, for each fixed } \lambda>0 ;(\alpha \in \mathbb{R}) .
$$

In the monograph [8] our restricted class for $\alpha \in \mathbb{R}$ is called of the "normalized regularly-varying functions" and shown to coincide with the "Zygmund class" ([8]; pp. $15,24)$ of those positive, measurable functions $f$ such that, for every $c>0$, “ $x^{-\alpha+c} f(x)$ is ultimately increasing" and " $x^{-\alpha-c} f(x)$ is ultimately decreasing". The Karamata class of regularly-varying functions at $+\infty$ coincides with the larger class of functions $f \in L_{\text {loc }}^{1}$, each defined on some neighborhood of $+\infty$ and asymptotically equivalent to a function regularly varying in the strong sense and we mention in passing that a convex $f$ satisfying (2.6) automatically satisfies (2.1): ([8]; \$1.11, no.13, p. 59) or ([10]; Th. 2.4 , p. 60). As far as a general class of rapidly-varying functions at $+\infty$ is concerned there are various options for which we refer to ([8]; $\$ 2.4$, pp. 83-86); anyway the smallest of these classes is still larger than the class of functions $f \in L_{l o c}^{1}$ asymptotically equivalent to a function rapidly varying in the strong sense, ([8]; Theorem 2.4.5, p. 86). The specification "at $+\infty$ " is not superfluous; the change of variable $x \mapsto\left|x-x_{0}\right|^{-1}$ allows the definition of the corresponding classes as $x \rightarrow x_{0}$. The restricted classes we have just defined are appropriate to define the concept of "variation of higher order" and suffice for many applications in the field of ordinary differential equations and asymptotic expansions. To visualize, notice that all infinitely-differentiable functions which can be represented as linear combinations, products, ratios and compositions of a finite number of powers, exponentials and logarithms as well as their derivatives of any order have principal parts at $+\infty$ which, as a rule, can be expressed by products of similar functions, hence such functions are strictly one-signed, strictly monotonic and strictly concave (or convex) on a neighborhood of $+\infty$ so that the quantity $x f^{\prime}(x) / f(x)$ is ultimately monotonic and the limit in (2.3) is granted.

3. Typical (indeed the most usual and useful) functions in $\mathcal{R}_{\alpha}(+\infty), \alpha \in \mathbb{R}$, are

$$
\phi_{\alpha, \beta_{k}, \gamma, c}(x):=x^{\alpha} \cdot\left[\prod_{k=1}^{n}\left(\ell_{k}(x)\right)^{\beta_{k}}\right] \cdot \exp \left(c(\log x)^{\gamma}\right),\left(\alpha, \beta_{k}, c \in \mathbb{R} ; 0<\gamma<1\right) .
$$


- Typical functions in $\mathcal{R}_{ \pm \infty}(+\infty)$ are

$$
\begin{gathered}
\psi_{\alpha, \beta_{k}, \gamma, \delta, c_{1}, c_{2}}(x):=x^{\alpha} \cdot\left[\prod_{k=1}^{n}\left(\ell_{k}(x)\right)^{\beta_{k}}\right] \cdot \exp \left(c_{1}(\log x)^{\gamma}\right) \cdot \exp \left(c_{2} x^{\delta}\right), \\
\left(\alpha, \beta_{k} \in \mathbb{R} ; \gamma>1 ; \delta>0 ; c_{1}^{2}+c_{2}^{2}>0\right),
\end{gathered}
$$

whose index of variation is: " $\left(\operatorname{sign} c_{2}\right) \cdot \infty$ ” if $c_{2} \neq 0$ or “ $\left(\operatorname{sign} c_{1}\right) \cdot \infty$ ” if $c_{2}=0$. If $c_{2} \neq 0, \gamma$ may be any number $>0$. Also notice that " $f \in \mathcal{R}_{-\infty}(+\infty)$ iff $1 / f \in \mathcal{R}_{+\infty}(+\infty)$ ".

- Typical monotonic functions in $\mathcal{R}_{0}(+\infty)$, besides the nonzero constants, are

$$
\left\{\begin{array}{l}
\left(\ell_{k}(x)\right)^{\beta_{k}},\left(k \geq 1, \beta_{k} \in \mathbb{R} \backslash 0\right) ; \\
\exp \left(c(\log x)^{\gamma}\right),(c \in \mathbb{R} \backslash 0,0<\gamma<1) ; \exp \left(c\left(\ell_{k}(x)\right)^{\gamma_{k}}\right),\left(c \in \mathbb{R} \backslash 0, k \geq 2, \gamma_{k}>0\right) ;
\end{array}\right.
$$

and their products

$$
\left[\prod_{k=1}^{n}\left(\ell_{k}(x)\right)^{\beta_{k}}\right] \cdot \exp \left[\sum_{h=1}^{m} c_{h}\left(\ell_{h}(x)\right)^{\gamma_{h}}\right],\left(\beta_{k}, c_{h} \in \mathbb{R}, 0<\gamma_{1}<1, \gamma_{h}>0 \text { for } h \geq 2\right) \text {. }
$$

Separating the cases $\alpha \neq 0$ and $\alpha=0$ we may rewrite (2.1) in the form

$$
\text { either } f^{\prime}(x) \sim \alpha x^{-1} f(x) \text { or } f^{\prime}(x)=o\left(x^{-1} f(x)\right), x \rightarrow+\infty \text {; }
$$

each of these may be viewed as an "asymptotic (ordinary) differential equation of first order" and it is easily shown (Proposition 2.1 below) that the solutions of the first one of them share the asymptotic properties of the solutions of the ordinary differential equation $f^{\prime}(x)=\alpha x^{-1} f(x)$. From the identity

$$
\begin{aligned}
& \log f(x)=C+\int_{T}^{x}\left[f^{\prime}(t) / f(t)\right] \mathrm{d} t \\
& \text { or equivalently } f(x)=\bar{C} \cdot \exp \left(\int_{T}^{x}\left[f^{\prime}(t) / f(t)\right] \mathrm{d} t\right), \bar{C} \neq 0,
\end{aligned}
$$

we get the characterization: An absolutely continuous function $f$ belongs to the class $\mathcal{R}_{\alpha}(+\infty), \alpha \in \mathbb{R}$, iff there exist two numbers $c \neq 0, T>0$ and a locally-integrable function $\epsilon(x)=o(1), x \rightarrow+\infty$, such that $f$ admits of the following representation:

$$
f(x)=c x^{\alpha} \cdot \exp \left(\int_{T}^{x}[\epsilon(t) / t] \mathrm{d} t\right), \forall x \geq T .
$$

And an analogous statement holds true for a rapidly-varying function with $\lim _{x \rightarrow+\infty} \epsilon(x)= \pm \infty$.

As a first rough asymptotic information:

$$
\begin{gathered}
f \in \mathcal{R}_{\alpha}(+\infty) \Rightarrow \log f(x)=\alpha \log x+o(\log x) \Rightarrow f(x)=\exp ([\alpha+o(1)] \cdot \log x) \equiv x^{\alpha+o(1)} \\
\quad f \in \mathcal{R}_{ \pm \infty}(+\infty) \Rightarrow f(x)=\exp (\omega(x) \cdot \log x) \text {, with } \lim _{x \rightarrow+\infty} \omega(x)= \pm \infty
\end{gathered}
$$

Notice that either representation " $\log f(x)=\alpha \log x+\Phi(x)$ " or " $f(x)=x^{\alpha} \Phi(x)$ ", with $\Phi(x)=O(1), \quad x \rightarrow+\infty$, does not imply $f \in \mathcal{R}_{\alpha}(+\infty)$ as shown by the counterexample of

$$
f(x):=x^{\alpha}(2+\sin \phi(x)), \phi \in A C[T,+\infty),
$$


which is in the class $\mathcal{R}_{\alpha}(+\infty)$ iff $\phi^{\prime}(x)=o\left(x^{-1}\right)$.

The general classes of regularly- or rapidly-varying functions enjoy many useful algebraic and analytic properties but it is not self-evident that the same is true for our restricted classes, in particular that they are closed with respect to various operations. In the next subsection we give a list of the main properties omitting those proofs which are quite elementary based on the property of the logarithmic derivative:

$$
D_{\ell}\left(\prod_{k=1}^{n} f_{k}(x)\right)=\sum_{k=1}^{n} D_{\ell}\left(f_{k}(x)\right) \text {. }
$$

\subsection{Basic Properties of Regularly- or Rapidly-Varying Functions}

Proposition 2.1. (Algebraic and asymptotic properties of regularly-varying functions). The following properties hold true:

(i) Factorization:

$$
f \in \mathcal{R}_{\alpha}(+\infty), \alpha \in \mathbb{R} \Leftrightarrow f(x) \equiv x^{\alpha} L(x) \text {, with } L \text { slowly varying. }
$$

(ii) Growth-order estimates:

$$
\begin{gathered}
f \in \mathcal{R}_{\alpha}(+\infty), \alpha \in \mathbb{R} \Rightarrow x^{\alpha-\epsilon} \ll f(x) \ll x^{\alpha+\epsilon}, x \rightarrow+\infty, \forall \epsilon>0 ; \\
L \text { slowly varying } \Rightarrow x^{-\epsilon} \ll L(x) \ll x^{\epsilon}, x \rightarrow+\infty, \forall \epsilon>0 ; \\
\lim _{x \rightarrow+\infty} f(x)= \begin{cases}0 & \text { if }-\infty \leq \alpha<0, \\
+\infty & \text { if } 0<\alpha \leq+\infty .\end{cases}
\end{gathered}
$$

But for $\alpha=0$ all the possible contingencies may occur for this limit as shown by the following functions of class $\left.\left.C^{\infty}\right] 1,+\infty\right)$ :

$$
\begin{aligned}
& \text { 1; }(\log x)^{\delta}, \delta \neq 0 ;+\sin \left((\log x)^{\delta}\right), c>1,0<\delta<1 ; \\
& \exp \left[(\log x)^{\delta} \cdot \sin \left((\log x)^{\delta}\right)\right], 0<\delta<1 / 2 .
\end{aligned}
$$

The third and the fourth of these functions are not ultimately monotonic. The third with bounded oscillations and the fourth, call it $f$, with unbounded oscillations. $\liminf _{x \rightarrow+\infty} f(x)=0, \limsup _{x \rightarrow+\infty} f(x)=+\infty$.

(iii) Algebraic operations. If $f \in \mathcal{R}_{\alpha}(+\infty), g \in \mathcal{R}_{\beta}(+\infty),(\alpha, \beta \in \mathbb{R})$ then the following functions are regularly varying as well with the specified index of variation $\delta$ :

$$
\begin{gathered}
{[f(x)]^{\gamma} \quad \text { with index } \delta=\alpha \cdot \gamma(\forall \gamma \in \mathbb{R}) ;} \\
f(x) \cdot g(x) \quad \text { with index } \delta=\alpha+\beta ; \\
f(x) / g(x) \quad \text { with index } \delta=\alpha-\beta ; \\
c f(x) \quad \text { with index } \delta=\alpha(c=\text { constant }>0) ; \\
c_{1} f(x)+c_{2} g(x) \quad \text { with index } \delta=\max (\alpha, \beta)\left(c_{i}=\text { constant }>0\right) .
\end{gathered}
$$

For $\alpha \neq \beta$ no restriction on the signs of the $c_{i}$ 's is necessary in (2.27), obviously not both zero.

(iv) Composition. If $f, g$ are as in (iii) and if $\lim _{x \rightarrow+\infty} g(x)=+\infty$ then to the 
above list we may add the composition

$$
f \circ g \text { with index } \delta=\alpha \cdot \beta \text {. }
$$

In particular, if $f \in \mathcal{R}_{\alpha}(+\infty), \alpha \in \mathbb{R}$, then $f \circ \log \in \mathcal{R}_{0}(+\infty)$; and if $f \in \mathcal{R}_{\alpha}(+\infty), \alpha \in \mathbb{R} \backslash\{0\}$, or if $f \in \mathcal{R}_{0}(+\infty)$ and $f(+\infty)=+\infty$, then $|\log f| \in \mathcal{R}_{0}(+\infty)$. In the case $\alpha=0$ it may happen that $|\log f|$ has no index of variation as shown by the third function in (2.22) for some values of the constant c. In fact as $x \rightarrow+\infty$ the function $\log \left(c+\sin \left[(\log x)^{\delta}\right]\right), 0<\delta<1$, changes sign infinitely often for $1<c<2$, has infinitely many zeros for $c=2$ and it is slowly varying for $c>2$.

(v) The particular case $\alpha=\beta=0$ in (iii) and (iv) states that if $f, g$ are slowly varying then so are the functions

$$
\left\{\begin{array}{l}
f^{\gamma},(\gamma \in \mathbb{R}) ; f \cdot g ; f / g ; c f,(c=\text { constant }>0) \\
c_{1} f+c_{2} g,\left(c_{i}=\text { constant }>0\right) ; f \circ g, \text { provided that } g(+\infty)=+\infty
\end{array}\right.
$$

The examples in (2.22) show that a pair $f, g \in \mathcal{R}_{0}(+\infty)$ may not be comparable at $+\infty$ meaning that one or both of the limits " $\lim f / g, \lim g / f$, as $x \rightarrow+\infty$, " may fail to exist in $\overline{\mathbb{R}}$. By the factorization in (2.18) the same applies to a pair $f, g \in \mathcal{R}_{\alpha}(+\infty)$.

(vi) Inversion. If $f \in \mathcal{R}_{\alpha}(+\infty), \alpha \in \mathbb{R} \backslash\{0\}$, then $f^{\prime}$ has ultimately one strict sign hence the restriction of $f$ to a suitable neighborhood of $+\infty$ has an inverse function $f^{-1}$; for $\alpha>0, f^{-1}$ is defined on a neighborhood of $+\infty$ as well and we have that

$$
f^{-1} \in \mathcal{R}_{\delta}(+\infty) \text { with } \delta=1 / \alpha .
$$

(vii) Asymptotic comparison. If $f \in \mathcal{R}_{\alpha}(+\infty)$ and $g \in \mathcal{R}_{\beta}(+\infty)$, with $\alpha, \beta \in \mathbb{R}$, then:

$$
\begin{gathered}
g(x) \begin{cases}\sim c f(x), x \rightarrow+\infty(c>0) & \Rightarrow \alpha=\beta ; \\
=o(f(x)), x \rightarrow+\infty & \Rightarrow \beta \leq \alpha ;\end{cases} \\
\alpha>\beta \Rightarrow f(x) \gg g(x), x \rightarrow+\infty,
\end{gathered}
$$

whereas no inference can be drawn if $\alpha=\beta$ as shown by the functions in (2.22).

Proof. (i) is trivial and (ii) follows from (2.13) as

$$
\forall \eta>0 \quad \exists T_{\eta} \geq T: x \geq T_{\eta} \Rightarrow\left|\int_{T}^{x}[\epsilon(t) / t] \mathrm{d} t\right| \leq \eta \log \frac{x}{T} .
$$

(iii) For the linear combination in (2.27) in the case $\alpha=\beta$ we have:

$$
\begin{aligned}
x\left(c_{1} f^{\prime}(x)+c_{2} g^{\prime}(x)\right) & =c_{1} \frac{x f^{\prime}(x)}{f(x)} f(x)+c_{2} \frac{x g^{\prime}(x)}{g(x)} g(x) \\
& =\alpha\left(c_{1} f(x)+c_{2} g(x)\right)+o\left(c_{1} f(x)\right)+o\left(c_{2} g(x)\right) \\
& =\cdots \text { by the positivity of } c_{1} f(x), c_{2} g(x) \cdots \\
& =\alpha\left(c_{1} f(x)+c_{2} g(x)\right)+o\left(c_{1} f(x)+c_{2} g(x)\right) .
\end{aligned}
$$

In the case $\alpha \neq \beta$, say $\alpha>\beta$, we have $g(x)=o(f(x))$ (see the proof of (2.32) below) and: 


$$
\begin{aligned}
\frac{x\left(c_{1} f^{\prime}(x)+c_{2} g^{\prime}(x)\right)}{c_{1} f(x)+c_{2} g(x)} & =\frac{\alpha c_{1} f(x)+\beta c_{2} g(x)+o\left(c_{1} f(x)\right)+o\left(c_{2} g(x)\right)}{c_{1} f(x)+c_{2} g(x)} \\
& =\frac{\alpha c_{1} f(x)+o(f(x))}{c_{1} f(x)+o(f(x))}=\alpha+o(1) .
\end{aligned}
$$

To prove (iv) write

$$
D_{\ell}(f(g(x)))=\frac{f^{\prime}(g(x)) g^{\prime}(x)}{f(g(x))}=\frac{f^{\prime}(g(x)) g(x)}{f(g(x))} \cdot \frac{g^{\prime}(x)}{g(x)},
$$

and notice that

$$
\left\{\begin{array}{l}
\lim _{x \rightarrow+\infty} f^{\prime}(g(x)) g(x) / f(g(x))=\lim _{y \rightarrow+\infty} y f^{\prime}(y) / f(y)=\alpha ; \\
g^{\prime}(x) / g(x)=\beta x^{-1}+o\left(x^{-1}\right) .
\end{array}\right.
$$

If $f \in \mathcal{R}_{\alpha}(+\infty), \alpha \neq 0$, then $\log f(x) \rightarrow \pm \infty$ as $x \rightarrow+\infty$ and $x(\log f(x))^{\prime} / \log f(x)=\left(x f^{\prime}(x) / f(x)\right) / \log f(x)=o(1)$.

To prove (vi) evaluate the following limit by the change of variable $x=f^{-1}(y)$ :

$$
\lim _{y \rightarrow+\infty} y\left(f^{-1}(y)\right)^{\prime} / f^{-1}(y) \equiv \lim _{y \rightarrow+\infty} \frac{y}{f^{-1}(y) \cdot f^{\prime}\left(f^{-1}(y)\right)}=\lim _{x \rightarrow+\infty} \frac{f(x)}{x f^{\prime}(x)}=1 / \alpha .
$$

Last: (2.32) follows from (2.25) and (2.21); relations in (2.31) follow from (2.32)

Remarks. 1. The properties in (iii), (iv) and (vi) are the same as those valid for the standard powers. The first inference in (2.31) can be interpreted by saying that the class $\mathcal{R}_{\alpha}(+\infty), \alpha$ fixed, is closed under the relation of "asymptotic equivalence" in the specified sense that any regularly-varying function asymptotically equivalent to a function in $\mathcal{R}_{\alpha}(+\infty)$ belongs to the same class; but it is false that "any function in $A C[T,+\infty)$ and asymptotically equivalent to a function in $\mathcal{R}_{\alpha}(+\infty)$ is in the same class" for the simple reason that it may not be regularly varying in the strong sense: counterexample of $f(x):=x^{\alpha}+\sin x, 0<\alpha \leq 1$. This shortcoming is overcome by the Karamata concept of regular variation.

2. In (2.27) it is essential that all the involved quantities (functions and constants) have one and the same sign for $\alpha=\beta$ otherwise possible cancellation of terms may yield any growth-order as shown, e.g., by $L_{1}(x)-L_{2}(x)$ where $L_{1}(x):=\log x$ and $L_{2}(x):=\log x+\phi(x)$ where $\phi$ is any of the three functions $x^{a}, a<0 ; \mathrm{e}^{-x} ; x^{a} \sin x, a<0$. The property in (2.27) is generalized in Proposition 2.3.

3. The "Zygmund property" cited after (2.6) and concerning the ultimate strict monotonicity of $x^{-\alpha \pm c} f(x), c>0$, is trivially checked for regular variation in the present strong sense by directly evaluating the derivative of this product and using (2.11).

4. A less direct proof of (2.27) uses the decomposition:

$$
\left\{\begin{array}{l}
\frac{\left(c_{1} f(x)+c_{2} g(x)\right)^{\prime}}{c_{1} f(x)+c_{2} g(x)} \equiv \frac{f^{\prime}(x)}{f(x)} \cdot H_{1}(x)+\frac{g^{\prime}(x)}{g(x)} H_{2}(x), \\
H_{1}(x):=\left(1+\frac{c_{2} g(x)}{c_{1} f(x)}\right)^{-1} ; \quad H_{2}(x):=\left(1+\frac{c_{1} f(x)}{c_{2} g(x)}\right)^{-1},
\end{array}\right.
$$


similar to a device which reveals efficient in the case of slow variation in the general weaker sense: see Seneta ([10], p. 19).

Examples. 1. Referring to the third function in (2.22) we mention that it can be proved that the function " $c+\sin \left((\log x)^{\delta}\right), c>1, \delta \geq 1$," is not slowly varying at $+\infty$ even in the general weak sense and the same is true for " $c+\sin \left(x^{\alpha}\right), \alpha>0$ ". The function " $x^{\alpha}+\sin x$ " is regularly varying (of index $\alpha$ ) in the general weak sense for each $\alpha>0$ for the simple reason that " $x^{\alpha}+\sin x \sim x^{\alpha}, x \rightarrow+\infty$ "; but it is regularly varying in the strong sense only for $\alpha>1$.

2. If $\phi \in A C[T,+\infty), \phi(+\infty)=+\infty$, then:

$$
\left\{\begin{array}{l}
c+\sin \phi(x), c>1, \in \mathcal{R}_{0}(+\infty) \Leftrightarrow \phi^{\prime}(x)=o\left(x^{-1}\right), x \rightarrow+\infty ; \\
c+(\sin \phi(x))^{2}, c>0, \in \mathcal{R}_{0}(+\infty) \Leftrightarrow \phi^{\prime}(x)=o\left(x^{-1}\right), x \rightarrow+\infty ; \\
x^{\alpha}+\sin \phi(x), \alpha>0, \in \mathcal{R}_{\alpha}(+\infty) \Leftrightarrow \phi^{\prime}(x)=o\left(x^{\alpha-1}\right), x \rightarrow+\infty .
\end{array}\right.
$$

3. The function $L_{-}(x):=\exp \left(-(\log x)^{\delta}\right), 0<\delta<1$, is slowly varying and tends to zero, as $x \rightarrow+\infty$, faster than any negative power of $\log x$; and the function $L_{+}(x):=\exp \left((\log x)^{\delta}\right), 0<\delta<1$, is slowly varying and diverges to $+\infty$ faster than any positive power of $\log x$.

Proposition 2.2. (Algebraic and asymptotic properties of rapidly-varying functions). The following properties hold true:

(i) Growth-order estimates:

$$
\left\{\begin{array}{l}
f \in \mathcal{R}_{+\infty}(+\infty) \Rightarrow f(x) \gg x^{\alpha}, x \rightarrow+\infty, \forall \alpha>0, \\
f \in \mathcal{R}_{-\infty}(+\infty) \Rightarrow f(x) \ll x^{-\alpha}, x \rightarrow+\infty, \forall \alpha>0,
\end{array}\right.
$$

(ii) Algebraic operations:

$$
\begin{gathered}
f \in \mathcal{R}_{+\infty}(+\infty) \Leftrightarrow(f(x))^{\gamma} \in \mathcal{R}_{+\infty}(+\infty) \text { if } \gamma>0 \\
\Leftrightarrow(f(x))^{\gamma} \in \mathcal{R}_{-\infty}(+\infty) \text { if } \gamma<0 . \\
\left\{\begin{array} { l } 
{ f \in \mathcal { R } _ { \alpha } ( + \infty ) , \alpha \in \mathbb { R } , } \\
{ g \in \mathcal { R } _ { \pm \infty } ( + \infty ) }
\end{array} \Rightarrow \left\{\begin{array}{l}
f \cdot g \in \mathcal{R}_{ \pm \infty}(+\infty) ;(f \cdot g) \sim f \cdot g^{\prime} ; \\
f / g \in \mathcal{R}_{+\infty}(+\infty) ; g / f \in \mathcal{R}_{ \pm \infty}(+\infty) .
\end{array}\right.\right. \\
\left\{\begin{array}{l}
f, g \in \mathcal{R}_{+\infty}(+\infty) \Rightarrow f \cdot g \in \mathcal{R}_{+\infty}(+\infty) ; \\
f, g \in \mathcal{R}_{-\infty}(+\infty) \Rightarrow f \cdot g \in \mathcal{R}_{-\infty}(+\infty) ;
\end{array}\right.
\end{gathered}
$$

with no inference about the quotient $f / g$ in (2.44) as shown by $\mathrm{e}^{c_{1} x} / \mathrm{e}^{c_{2} x}$ in the three cases " $c_{1}<,=,>c_{2}$ " and by $\mathrm{e}^{x}(3+\sin x) / \mathrm{e}^{x}$. Together with the result in (2.25) we may assert that: For any two functions " $f \in \mathcal{R}_{\alpha}(+\infty), g \in \mathcal{R}_{\beta}(+\infty)$," with " $\alpha, \beta \in \overline{\mathbb{R}}$, $\alpha<\beta$," the following limits as $x \rightarrow+\infty$ hold true:

$\lim f / g=0, \lim g / f=+\infty$.

(iii) Compositions:

$$
\left\{\begin{array}{l}
f \in \mathcal{R}_{\alpha}(+\infty), \alpha>0, g \in \mathcal{R}_{+\infty}(+\infty) \Rightarrow f \circ g \in \mathcal{R}_{+\infty}(+\infty) ; \\
f \in \mathcal{R}_{\alpha}(+\infty), \alpha<0, g \in \mathcal{R}_{+\infty}(+\infty) \Rightarrow f \circ g \in \mathcal{R}_{-\infty}(+\infty) ; \\
f \in \mathcal{R}_{ \pm \infty}(+\infty), g \in \mathcal{R}_{\alpha}(+\infty), \alpha>0 \Rightarrow f \circ g \in \mathcal{R}_{ \pm \infty}(+\infty) ; \\
f \in \mathcal{R}_{ \pm \infty}(+\infty), g \in \mathcal{R}_{+\infty}(+\infty) \Rightarrow f \circ g \in \mathcal{R}_{ \pm \infty}(+\infty) ;
\end{array}\right.
$$


with no inference about $f \circ g$ in case " $f \in \mathcal{R}_{ \pm \infty}(+\infty), g \in \mathcal{R}_{0}(+\infty)$ " or viceversa as shown by

$$
\ell_{2}\left(\mathrm{e}^{x}\right)=\log x ; \log \left(\exp \left(x^{\alpha}\right)\right)=x^{\alpha} ; \log \left(\exp _{2}(x)\right)=\mathrm{e}^{x}
$$

In particular

$$
f \in \mathcal{R}_{\alpha}(+\infty), \alpha \neq 0, \begin{cases}\exp f \in \mathcal{R}_{+\infty}(+\infty) & \text { if } \alpha>0, \\ \exp f \in \mathcal{R}_{0}(+\infty) & \text { if } \alpha<0 ;(\exp f=1+o(1)) .\end{cases}
$$

(iv) Inversions. Roughly speaking the inverse of a rapidly-varying function is slowly varying and viceversa. To be precise, if $f \in \mathcal{R}_{+\infty}(+\infty)$ then its inverse $f^{-1}$ is defined on a suitable neighborhood of $+\infty$ and $f^{-1} \in \mathcal{R}_{0}(+\infty)$. Viceversa, if

$$
f \in \mathcal{R}_{0}(+\infty), f(+\infty)=+\infty, f \text { ultimately strictly increasing, }
$$

then $f^{-1} \in \mathcal{R}_{+\infty}(+\infty)$.

Proof. For the first three groups of relations we write down the proof only for $(f g)^{\prime} \sim f g^{\prime}$ in (2.43):

$$
(f g)^{\prime}=f g^{\prime} \cdot\left(1+\frac{f^{\prime} g}{f g^{\prime}}\right)=f g^{\prime} \cdot(1+\underbrace{\frac{x f^{\prime}}{f}}_{=\alpha+o(1)} \underbrace{\frac{g}{x g^{\prime}}}_{o(1)}) \sim f g^{\prime} .
$$

Both claims in (iv) are proved as in (2.38). $\square$

Examples about rapid variation in weak or strong sense. Comparing with the examples preceding Proposition 2.2 the function " $f(x):=\mathrm{e}^{x}+\sin \phi(x)$ " is rapidly varying in the general weak sense for any measurable $\phi$ because " $\mathrm{e}^{x}+\sin \phi(x) \sim \mathrm{e}^{x}, x \rightarrow+\infty$ ". Under the additional assumptions " $\phi \in A C[T,+\infty), \phi^{\prime}(x)=o\left(\mathrm{e}^{x}\right), x \rightarrow+\infty$, " then $f$ is rapidly varying in the strong sense with index $+\infty$.

Quite differently, if “ $\phi \in A C[T,+\infty), \phi(+\infty)=+\infty$ ” then " $\mathrm{e}^{-x}+\sin \phi(x) \notin \mathcal{R}_{\alpha}(+\infty)$ " for any value of $\alpha \in \overline{\mathbb{R}}$ because it changes sign infintely often; and the function $g(x):=\mathrm{e}^{-x}+(\sin \phi(x))^{2}$, though strictly positive, has no limit at $+\infty$, hence “ $g \notin \mathcal{R}_{\alpha}(+\infty)$ ” for any value of $\alpha \in \overline{\mathbb{R}} \backslash\{0\}$. Moreover, the explicit expression of

$$
\frac{x g^{\prime}(x)}{g(x)}=-x \frac{\mathrm{e}^{-x}-2 \phi^{\prime}(x) \sin \phi(x) \cos \phi(x)}{\mathrm{e}^{-x}+(\sin \phi(x))^{2}}
$$

also shows that " $g \notin \mathcal{R}_{0}(+\infty)$ " because " $\sin \phi\left(x_{n}\right)=0 \Rightarrow \lim _{n \rightarrow \infty} x_{n} g^{\prime}\left(x_{n}\right) / g\left(x_{n}\right)=-\infty$ " whereas “ $\cos \phi\left(y_{n}\right)=0 \Rightarrow \lim _{n \rightarrow \infty} y_{n} g^{\prime}\left(y_{n}\right) / g\left(y_{n}\right)=0$ ”.

In the next proposition we collect various results about linear combinations, results particularly useful in asymptotic contexts.

Proposition 2.3. (I) (Positive linear combinations of different types of asymptotic variations).

$$
f \in \mathcal{R}_{ \pm \infty}(+\infty) \Leftrightarrow c f \in \mathcal{R}_{ \pm \infty}(+\infty) \text { if } c=\text { constant }>0 .
$$

If $c_{1}, c_{2}$ are positive constants then: 


$$
\left\{\begin{array}{l}
f, g \in \mathcal{R}_{+\infty}(+\infty) \Rightarrow c_{1} f+c_{2} g \in \mathcal{R}_{+\infty}(+\infty) \\
f, g \in \mathcal{R}_{-\infty}(+\infty) \Rightarrow c_{1} f+c_{2} g \in \mathcal{R}_{-\infty}(+\infty) \\
f \in \mathcal{R}_{\alpha}(+\infty), \alpha \in \mathbb{R}, g \in \mathcal{R}_{+\infty}(+\infty) \Rightarrow c_{1} f+c_{2} g \in \mathcal{R}_{+\infty}(+\infty)
\end{array}\right.
$$

without any further restriction on $f, g$. On the contrary, we can prove the following two inferences only under one of the two specified restrictions.

$$
\left\{\begin{array}{l}
f \in \mathcal{R}_{-\infty}(+\infty), g \in \mathcal{R}_{\alpha}(+\infty), \alpha \in \mathbb{R} \Rightarrow c_{1} f+c_{2} g \in \mathcal{R}_{\alpha}(+\infty) ; \\
f \in \mathcal{R}_{-\infty}(+\infty), g \in \mathcal{R}_{+\infty}(+\infty) \Rightarrow c_{1} f+c_{2} g \in \mathcal{R}_{+\infty}(+\infty)
\end{array}\right.
$$

provided that:

$$
\text { either } f^{\prime}(x)=o\left(g^{\prime}(x)\right) \text { or } f^{\prime} \text { ultimately monotonic. }
$$

These inferences, together with (2.27), are summarized in

$$
\left\{f \in \mathcal{R}_{\alpha}(+\infty), g \in \mathcal{R}_{\beta}(+\infty)\right\} \Rightarrow c_{1} f+c_{2} g \in \mathcal{R}_{\max (\alpha, \beta)}(+\infty),
$$

whatever the positive constants $c_{1}, c_{2}$ and the extended real numbers $\alpha, \beta \in \overline{\mathbb{R}}$ except for the two cases in (2.53) wherein a restriction is added: See a discussion after the proof.

(II) (Arbitrary linear combinations of asymptotic scales). Let the functions $\phi_{i} \in A C[T,+\infty), i=1, \cdots, n$, form the scale

$$
\phi_{1}(x) \gg \phi_{2}(x) \gg \cdots \gg \phi_{n}(x), x \rightarrow+\infty,
$$

and let one of the following conditions be satisfied, either $\phi_{1}(x) \neq 0 \forall x$ large enough and

$$
\left\{\begin{array}{l}
\phi_{i}^{\prime}(x)=o\left(\phi_{1}^{\prime}(x)\right), x \rightarrow+\infty, \forall i \geq 2 ; \text { in particular } \\
\phi_{1}^{\prime}(x) \gg \phi_{2}^{\prime}(x) \gg \cdots \gg \phi_{n}^{\prime}(x), x \rightarrow+\infty
\end{array}\right.
$$

or $\phi_{i}(x) \neq 0 \forall x$ large enough and $\forall i$, and

$$
\left\{\begin{array}{l}
\phi_{i}^{\prime}(x) / \phi_{i}(x)=O\left(\phi_{1}^{\prime}(x) / \phi_{1}(x)\right), x \rightarrow+\infty, \forall i \geq 2 ; \text { in particular } \\
\phi_{1}^{\prime}(x) / \phi_{1}(x) \succcurlyeq \phi_{2}^{\prime}(x) / \phi_{2}(x) \succcurlyeq \cdots \succcurlyeq \phi_{n}^{\prime}(x) / \phi_{n}(x), x \rightarrow+\infty ;
\end{array}\right.
$$

with $f(x) \succcurlyeq g(x)$ meaning $g(x)=O(f(x))$. Then

$$
D_{\ell}\left(\sum_{i=1}^{n} c_{i} \phi_{i}(x)\right) \sim D_{\ell}\left(\phi_{1}(x)\right), x \rightarrow+\infty\left(c_{i} \in \mathbb{R}, c_{1} \neq 0\right),
$$

so that: if $\phi_{1}$ has an index of variation at $+\infty$ in the strong sense also the function $\left|\sum_{i=1}^{n} c_{i} \phi_{i}(x)\right|$ has the same index of variation. Without both additional conditions (2.57)-(2.58) there is no general claim about the type of growth of $\sum_{i} c_{i} \phi_{i}$ as shown by simple counterexamples reported after the proof.

(III) If

$$
\phi_{i} \in \mathcal{R}_{\alpha_{i}}(+\infty), 1 \leq i \leq n, \alpha_{i} \in \mathbb{R} ; \phi_{1}(x) \gg \phi_{2}(x) \gg \cdots \gg \phi_{n}(x), x \rightarrow+\infty,
$$

then $\left|\sum_{i=1}^{n} c_{i} \phi_{i}(x)\right| \in \mathcal{R}_{\alpha_{1}}(+\infty) \forall c_{i} \in \mathbb{R}$. If $\alpha_{1}>\cdots>\alpha_{n}$ then $\left(\phi_{1}, \cdots, \phi_{n}\right)$ is automatically an asymptotic scale. 
(IV) Besides (2.56) let

$$
\psi_{i} \in \mathcal{R}_{\beta_{i}}(+\infty), 1 \leq i \leq m ; \psi_{1}(x) \gg \psi_{2}(x) \gg \cdots \gg \psi_{m}(x), x \rightarrow+\infty,
$$

and put

$$
R(x):=\frac{a_{1} \phi_{1}(x)+\cdots+a_{n} \phi_{n}(x)}{b_{1} \psi_{1}(x)+\cdots+b_{m} \psi_{m}(x)},\left(a_{i}, b_{i} \in \mathbb{R} ; a_{1}, b_{1} \neq 0\right) .
$$

Then $|R| \in \mathcal{R}_{\alpha_{1}-\beta_{1}}(+\infty)$. In particular

$$
\sum_{i=1}^{n} a_{i} x^{\alpha_{i}}\left(\ell_{i}(x)\right)^{\gamma_{i}} / \sum_{i=1}^{m} b_{i} x^{\beta_{i}}\left(\ell_{i}(x)\right)^{\delta_{i}} \in \mathcal{R}_{\delta}(+\infty),\left(a_{i}, b_{i}>0 ; \alpha_{i}, \beta_{i}, \gamma_{i}, \delta_{i} \in \mathbb{R}\right),
$$

with $\delta=\max \alpha_{i}-\max \beta_{i}$.

Proof. We may include the constants $c_{i}$ into the functions $f, g$. For the first two inferences in (2.52) the assumptions imply that $\forall M>0 \exists T_{M} \geq T$ :

$$
x\left(f^{\prime}(x)+g^{\prime}(x)\right)=\frac{x f^{\prime}(x)}{f(x)} f(x)+\frac{x g^{\prime}(x)}{g(x)} g(x)\left\{\begin{array}{l}
\geq M(f(x)+g(x)) \\
\leq-M(f(x)+g(x))
\end{array} \forall x \geq T_{M},\right.
$$

respectively for the first and the second inference and the claims follow. For the third inference in (2.52) we have " $f=o(g)$ " and " $x f^{\prime}=O(f)=o(g)$ ", hence:

$$
\frac{x\left(f^{\prime}(x)+g^{\prime}(x)\right)}{f(x)+g(x)}=\frac{o(g)+x g^{\prime}(x)}{g(x)[1+o(1)]}=\frac{x g^{\prime}(x)}{g(x)}[1+o(1)]+o(1) \rightarrow+\infty, x \rightarrow+\infty \text {. }
$$

A different elementary proof is achieved writing:

$$
\frac{f^{\prime}}{g^{\prime}}=(\underbrace{\frac{x f^{\prime}}{f}}_{\alpha+o(1)} \cdot \underbrace{\frac{g}{x g^{\prime}}}_{o(1)}) \cdot \frac{f}{g}=o\left(\frac{f}{g}\right)=o(1) ;
$$

hence $f^{\prime}=o\left(g^{\prime}\right)$. So we get $x\left(f^{\prime}+g^{\prime}\right) /(f+g) \sim\left(x g^{\prime} / g\right)$; and this is a special case of the result in part (II). The two inferences in (2.53) follow from part (II) under condition $f^{\prime}=o\left(g^{\prime}\right)$. Under condition " $f^{\prime}$ ultimately monotonic" an argument runs as follows: the assumptions in both cases imply " $f / g \equiv f \cdot(1 / g) \in \mathcal{R}_{-\infty}(+\infty)$ ", see Proposition 2.2-(ii), hence " $f(x)=o\left(x^{-\delta} g(x)\right) \forall \delta>0$ " so that " $f+g \sim g$ ". Moreover it will be proved in Proposition 2.5 that the monotonicity of $f^{\prime}$ implies its satisfying the same asymptotic estimates as $f$ in (2.41) i.e. " $f^{\prime}(x)=o\left(x^{-\delta}\right) \forall \delta>0$ ". Now we have:

$$
\frac{x\left(f^{\prime}(x)+g^{\prime}(x)\right)}{f(x)+g(x)} \sim \frac{x f^{\prime}(x)+x g^{\prime}(x)}{g(x)}=\frac{x f^{\prime}(x)}{g(x)}+\frac{x g^{\prime}(x)}{g(x)} \sim \frac{x g^{\prime}(x)}{g(x)}
$$

because we shall prove in a moment that " $x f^{\prime}(x) / g(x)=o(1)$ ":

$$
\left\{\begin{array}{l}
g \in \mathcal{R}_{\alpha}(+\infty) \stackrel{\text { by }(2.19)}{\Rightarrow} g(x) \gg x^{\alpha-\epsilon} \Rightarrow x f^{\prime}(x) / g(x) \ll x^{\epsilon-\alpha-\delta} \forall \epsilon, \delta>0 ; \\
g \in \mathcal{R}_{+\infty}(+\infty) \stackrel{\text { by }(2.41)}{\Rightarrow} 1 / g(x)=o(1) \Rightarrow x f^{\prime}(x) / g(x)=o(1) .
\end{array}\right.
$$

In part (II) the result involving (2.57) trivially follows from factoring out $c_{1} \phi_{1}$ and 
$c_{1} \phi_{1}^{\prime}$ in the left-hand side in (2.59), whereas to prove (2.59) under (2.58) we have first to notice that

$$
\sum_{i=2}^{n} c_{i} \phi_{i}^{\prime}=\sum_{i=2}^{n} c_{i} \frac{\phi_{i}^{\prime}}{\phi_{i}} \phi_{i}=\sum_{i=2}^{n} O\left(\frac{\phi_{1}^{\prime}}{\phi_{1}}\right) \cdot o\left(\phi_{1}\right)=o\left(\phi_{1}^{\prime}\right) .
$$

Claims in parts (III), (IV) are corollaries of the result in part (II) involving (2.58). $\square$

Remarks. 1. Using the decomposition in (2.39) the first two inferences in (2.52) may be proved with the restriction " $f=O(g)$ ". The trivial device in (2.66) would work well also under the assumptions: $f \in \mathcal{R}_{\alpha}(+\infty), g \in \mathcal{R}_{\beta}(+\infty) ; \alpha, \beta \in \mathbb{R} ; \beta \neq 0 \quad$ (which grants $\left.g^{\prime} \neq 0\right)$. This last restriction is overcome in the different proof provided for (2.27) as well as in an alternative more elaborated proof involving decomposition (2.39).

2. Conditions in (2.57) and (2.58) are independent. Any pair $f, g$ where $f$ is any function of type in (2.7) and $g$ is any function of type in (2.8) with $c_{2}<0$, hence $g \in \mathcal{R}_{-\infty}(+\infty)$, is such that: $f \ll g, f^{\prime} \ll g^{\prime}$ but $f^{\prime} / f \gg g^{\prime} / g$ : and this shows that (2.56)-(2.57) do not imply (2.58). And here is a pair satisfying a stronger relation than in (2.58) though relations in (2.56)-(2.57) dramatically fail:

$$
\left\{\begin{array}{l}
f(x):=\exp \left[x+x^{\alpha} \sin \left(x^{\beta}\right)\right], g(x):=\exp \left[x+x^{\alpha} \cos \left(x^{\beta}\right)\right], 0<\alpha<1,0<\beta<1-\alpha ; \\
\liminf _{x \rightarrow+\infty}(f(x) / g(x))=\liminf _{x \rightarrow+\infty}\left(f^{\prime}(x) / g^{\prime}(x)\right)=0 ; \\
\limsup _{x \rightarrow+\infty}(g(x) / f(x))=\limsup _{x \rightarrow+\infty}\left(g^{\prime}(x) / f^{\prime}(x)\right)=+\infty \\
\left.f^{\prime}(x) / f(x) \sim g^{\prime}(x) / g(x) \sim 1 \text { (hence } f, g \in \mathcal{R}_{+\infty}(+\infty)\right) .
\end{array}\right.
$$

Counterexamples concerning suppression of conditions (2.57)-(2.58). In the following we use three pairs of functions in ([4]; (9.12), (9.13), (9.14); p. 487):

$$
\begin{aligned}
& \left\{\begin{array}{l}
1+x^{-1} \gg a x^{-1},(a \neq 0) ; \phi_{1}^{\prime}(x) / \phi_{2}^{\prime}(x) \sim a^{-1} ; \phi_{2}^{\prime}(x) / \phi_{2}(x) \gg \phi_{1}^{\prime}(x) / \phi_{1}(x) ; \\
D_{\ell}\left(\phi_{1}+\phi_{2}\right) \sim(1+a) a^{-1} \phi_{2}^{\prime}=(1+a) x^{-1} \phi_{2}^{\prime} / \phi_{2} \text { if } a \neq-1 ; D_{\ell}\left(\phi_{1}+\phi_{2}\right) \equiv 0 \text { if } a=-1 ;
\end{array}\right. \\
& \left\{\begin{array}{l}
1+x^{-2} \gg a x^{-1},(a \neq 0) ; \phi_{1}^{\prime}(x) / \phi_{2}^{\prime}(x)=o(1) ; \phi_{2}^{\prime}(x) / \phi_{2}(x) \gg \phi_{1}^{\prime}(x) / \phi_{1}(x) ; \\
D_{\ell}\left(\phi_{1}+\phi_{2}\right) \sim \phi_{2}^{\prime}=a x^{-1} \phi_{2}^{\prime} / \phi_{2}
\end{array}\right.
\end{aligned}
$$

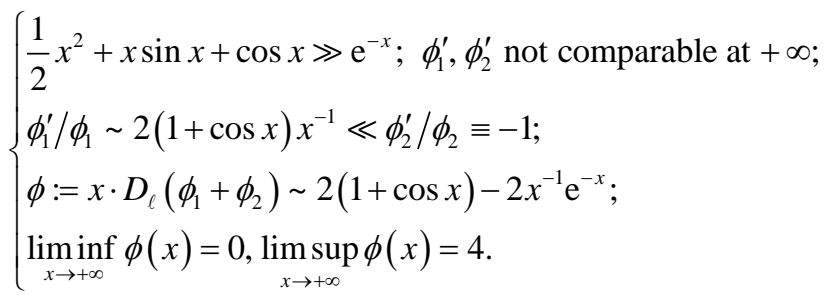

In the last example the function $\phi_{1}(x):=(1 / 2) x^{2}+x \sin x+\cos x \notin \mathcal{R}(+\infty)$ though it is regularly varying of index 2 in Karamata'e sense.

3. As concerns an additional restriction in the two inferences in (2.53) we have already remarked that the assumptions imply " $f+g \sim g$ ", hence if $f+g$ has an index of variation at $+\infty$ this must equal the index of $g$. We do not know whether inferences in (2.53) can be proved without any restriction or not. A possible counterexample with 
“ $g \in \mathcal{R}_{0}(+\infty), g(x) \sim 1$," and based on the same calculations in (2.67) would be provided by a function $f$ such that:

$$
f \in \mathcal{R}_{-\infty}(+\infty) ; f^{\prime}(+\infty) \text { does not exist as a finite number. }
$$

But for the time being we do not know any such function: see Proposition 2.5-(III).

For integrals of functions in our classes we report the classical results (with elementary proofs) to highlight a difference between the two cases.

Proposition 2.4. (I) (Integrals of regularly-varying functions). Let $f \in \mathcal{R}_{\alpha}(+\infty)$, $\alpha \in \mathbb{R}$, and $f>0$ on a given interval $[T,+\infty)$. Then:

$$
\begin{aligned}
& \alpha>-1 \Rightarrow\left\{\int^{+\infty} f=+\infty \text { and } \int_{T}^{x} f \in \mathcal{R}_{\alpha+1}(+\infty)\right\} ; \\
& \alpha<-1 \Rightarrow\left\{\int^{+\infty} f<+\infty \text { and } \int_{x}^{+\infty} f \in \mathcal{R}_{\alpha+1}(+\infty)\right\} ; \\
& \left\{\alpha=-1 \text { and } \int^{+\infty} f=+\infty\right\} \Rightarrow \int_{T}^{x} f \in \mathcal{R}_{0}(+\infty) ; \\
& \left\{\alpha=-1 \text { and } \int^{+\infty} f<+\infty\right\} \Rightarrow \int_{x}^{+\infty} f \in \mathcal{R}_{0}(+\infty) .
\end{aligned}
$$

The inferences in (2.74)-(2.75) are respectively equivalent to the following asymptotic relations expressing the behavior of the integral $\int^{x} f$ in terms of $f$ :

$$
\begin{aligned}
& f \in \mathcal{R}_{\alpha}(+\infty),-1<\alpha<+\infty \Rightarrow \int_{T}^{x} f \sim \frac{x f(x)}{\alpha+1} ; \\
& f \in \mathcal{R}_{\alpha}(+\infty),-\infty<\alpha<-1 \Rightarrow \int_{x}^{+\infty} f \sim \frac{x f(x)}{|\alpha+1|} .
\end{aligned}
$$

In the two cases (2.76)-(2.77) we may only assert, generally speaking, that

$$
\lim _{x \rightarrow+\infty} \int_{T_{0}}^{x} f / x f(x)=+\infty \text {, with the proper choice of } T_{0}: T_{0}=T \text { or } T_{0}=+\infty .
$$

(II) (Integrals of rapidly-varying functions). We have the rough estimates:

$$
\begin{aligned}
& f \in \mathcal{R}_{+\infty}(+\infty) \Rightarrow \int_{T}^{x} f \in \mathcal{R}_{+\infty}(+\infty) \text { i.e. } \int_{T}^{x} f=o(x f(x)), x \rightarrow+\infty \\
& f \in \mathcal{R}_{-\infty}(+\infty) \Rightarrow \int_{x}^{+\infty} f \in \mathcal{R}_{-\infty}(+\infty) \text { i.e. } \int_{x}^{+\infty} f=o(x f(x)), x \rightarrow+\infty .
\end{aligned}
$$

But under the stronger assumption $f \in A C^{1}[T,+\infty)$ and

$$
f(x)>0, f^{\prime}(x) \neq 0 \forall x ; f(x) / f^{\prime}(x)=o(x),\left(f(x) / f^{\prime}(x)\right)^{\prime}=o(1), x \rightarrow+\infty ;
$$

we have the exact principal parts.

$$
\begin{gathered}
f \in \mathcal{R}_{+\infty}(+\infty) \Rightarrow \int_{T}^{x} f \sim(f(x))^{2} / f^{\prime}(x) \equiv f(x) / D_{\ell} f(x), x \rightarrow+\infty \\
f \in \mathcal{R}_{-\infty}(+\infty) \Rightarrow \int_{x}^{+\infty} f \sim(f(x))^{2} /\left|f^{\prime}(x)\right| \equiv-f(x) / D_{\ell} f(x), x \rightarrow+\infty .
\end{gathered}
$$

Remarks. Notice that the formal rule in (2.84)-(2.85) does not coincide with that in (2.78)-(2.79) in accordance with relations in (2.104) below. If $f \in \mathcal{R}_{ \pm \infty}(+\infty)$ and $F$ is an antiderivative of its then the $\lim _{x \rightarrow+\infty} F(x) / f(x)$ may have any value depending on the behavior of $D_{\ell} f(x)$, and this gives rise to three concepts of "exponential variation" studied in Part II, $₫ 8$, of this paper.

Proof. The convergence or divergence of the integral in case $\alpha \neq 1$ trivially follows 
from the estimates in (2.19). (I) In the first and third cases one applies L'Hospital's rule to $\lim _{x \rightarrow+\infty} x f(x) / \int_{T}^{x} f$. In the second and fourth cases one applies L'Hospital's rule to $\lim _{x \rightarrow+\infty}-x f(x) / \int_{x}^{+\infty} f$, preliminarly noticing that $x f(x)=o(1), x \rightarrow+\infty$. This last relation follows from (2.19) in the second case whereas, in the fourth case, we have that

$$
f^{\prime}(x) / f(x) \sim-1 / x \Rightarrow \text { " } f^{\prime}(x)<0 \text { ultimately" }
$$

and this, in turn, together with condition $\int^{+\infty} f<+\infty$, implies the relation in question by the simple inequality:

$$
0<\frac{x}{2} f(x)<\int_{x / 2}^{x} f=o(1) ;
$$

see Pólya-Szegö ([11]; Part II, Chap. 3, Problem 113, pp. 77, 261). (II) The inferences in (2.81)-(2.82) directly follow from (2.2); for (2.81) we have

$$
\lim _{x \rightarrow+\infty} x f(x) / \int_{T}^{x} f \stackrel{H}{=} 1+\lim _{x \rightarrow+\infty} x f^{\prime}(x) / f(x)=+\infty,
$$

and analogously for (2.82). To prove (2.84) just write

$$
\int_{T}^{x} f(t) \mathrm{d} t \equiv \int_{T}^{x} \frac{f(t)}{f^{\prime}(t)} \mathrm{d} f(t)=c+\frac{f^{2}(x)}{f^{\prime}(x)}-\int_{T}^{x} f(t) \cdot\left(f(t) / f^{\prime}(t)\right) \mathrm{d} t,
$$

with a suitable constant $c$. Using the third condition in (2.83) we get

$$
\left(\int_{T}^{x} f\right)[1+o(1)]=c+\frac{f^{2}(x)}{f^{\prime}(x)}
$$

The divergence of $\int^{+\infty} f$ is granted by $f(+\infty)=+\infty$ and (2.84) follows. Analogous proof for (2.85) first noticing that, in this case, the second growth-estimate in (2.41) implies the convergence of both the first and the last integral in (2.87) as $x \rightarrow+\infty$, hence the convergence of $f^{2}(x) / f^{\prime}(x) ;(2.85)$ then follows by a similar integration by parts in $\int_{x}^{+\infty} f$

Remarks. 1. There is a difference between the two cases: though the character of regular or rapid variation of an antiderivative is elementarily checked, a useful result about the asymptotic behaviors in the rapid-variation case requires a restrictive assumption. Condition $f(x) / f^{\prime}(x)=o(x)$ in (2.83) directly follows from (2.2) whereas the additional asssumption means that this asymptotic relation is formally differentiable once. The following counterexample shows that it is not easy to get rid of a condition like this even if the second condition in (2.83) is replaced by the stronger one $f(x) / f^{\prime}(x) \asymp 1, x \rightarrow+\infty$, meaning that $0<c_{1} \leq\left|f(x) / f^{\prime}(x)\right| \leq c_{2}<+\infty$ for $x$ large enough:

$$
\left\{\begin{array}{l}
f_{1}(x):=(3+\cos x) \cdot \exp (3 x+\sin x) \\
f_{1}^{\prime}(x)=\left(9+\cos ^{2} x+6 \cos x-\sin x\right) \cdot \exp (3 x+\sin x) ; \\
f_{1}(x) / f_{1}^{\prime}(x) \asymp 1, x \rightarrow+\infty ;\left(f_{1}(x) / f_{1}^{\prime}(x)\right)^{\prime} \neq o(1), x \rightarrow+\infty ; \\
\int_{0}^{x} f_{1}=\exp (3 x+\sin x)-1 .
\end{array}\right.
$$


Here: $f_{1} \in \mathcal{R}_{+\infty}(+\infty) ; \quad f_{1}, f_{1}^{\prime}$ are strictly positive on $[1,+\infty) ; \quad F(x):=\int_{0}^{x} f_{1}$ is strictly positive, increasing and convex on $[1,+\infty)$ but the thesis in $(2.84)$ does not hold true because the oscillating factors appearing in the expressions of F,F',F" are not comparable between each other though they are very small when compared to the exponential factor $\mathrm{e}^{3 x}$. Neither is the additional asssumption necessary for the inferences (2.84)-(2.85): Proposition 8.2 in Part II of this work exhibits a very special subclass of rapidly-varying functions for which the asymptotic relations in (2.84)-(2.85) hold true without condition $\left(f / f^{\prime}\right)^{\prime}=o(1)$. An example similar to that in (2.89) and with $f / f^{\prime} \sim 1$ is reported after the proof of Proposition 4.2 to illustrate a different phenomenon.

2. The proof based on the device in (2.87) could be adapted to the case of a regularly-varying function observing that, for $\alpha \neq 0,(2.1)$ is equivalently expressed as

$$
f(x) / f^{\prime}(x)=\alpha^{-1} x+o(x), x \rightarrow+\infty,
$$

and imposing the extra-assumption of formal differentiation of this last relation, i.e.

$$
\left(f(x) / f^{\prime}(x)\right)^{\prime}=\alpha^{-1}+o(1), x \rightarrow+\infty .
$$

One would re obtain the inferences in (2.74)-(2.75) but under the unnecessary restrictions: (2.91) and $\alpha \neq 0$. Hence the device in (2.87) is unnatural for a regularly-varying $f$ whereas it is appropriate to the rapidly-varying case.

\subsection{Properties of the First Derivative}

The following properties of the first derivative are essential to develop the theory of higher-order variation.

Proposition 2.5. (Elementary asymptotic properties of the first derivative). The following hold true with all asymptotic properties referring to $x \rightarrow+\infty$.

(I) (Regular variation). The estimates in (2.19) imply that:

$$
\begin{gathered}
f^{\prime}(x) / f(x) \sim \alpha x^{-1},(\alpha>0) \Rightarrow\left\{\begin{array}{l}
f^{\prime}(x)>0 \text { ultimately, } \\
f^{\prime}(x) \sim \alpha x^{-1} f(x) \begin{cases}\rightarrow+\infty & \text { if } \alpha>1, \\
\rightarrow 0 & \text { if } 0<\alpha<1 ;\end{cases}
\end{array}\right. \\
f^{\prime}(x) / f(x) \sim \alpha x^{-1},(\alpha<0) \Rightarrow\left\{\begin{array}{l}
f^{\prime}(x)<0 \text { ultimately, } \\
f^{\prime}(x) \sim \alpha x^{-1} f(x)=o\left(x^{-1}\right) .
\end{array}\right.
\end{gathered}
$$

For $\alpha=1$ any circumstance is possible for $\lim _{x \rightarrow+\infty} f^{\prime}(x)$ as shown by

$$
\begin{gathered}
f_{1}(x):=x(\log x)^{\delta}, \delta>0, f_{1}^{\prime}(x) \rightarrow+\infty ; f_{2}(x):=x(\log x)^{\delta}, \delta<0, f_{2}^{\prime}(x) \rightarrow 0 ; \\
\left\{\begin{array}{l}
f_{3}(x):=x\left(2+\sin \left[(\log x)^{\delta}\right]\right),(0<\delta<1), \\
f_{3}^{\prime}(x)=2+\sin \left[(\log x)^{\delta}\right]+o(1), f_{3}^{\prime} \text { bounded but nonconvergent; }
\end{array}\right. \\
\left\{\begin{array}{l}
f_{4}(x):=\int_{1}^{x} \exp \left[(\log t)^{\delta} \cdot \sin \left((\log t)^{\delta}\right)\right] \mathrm{d} t, 0<\delta<1 / 2, \\
f_{4}^{\prime} \text { unbounded but nondivergent : see }(2.22) .
\end{array}\right.
\end{gathered}
$$


Moreover, for each $\alpha \neq 0$ we can exhibit an " $f \in \mathcal{R}_{\alpha}(+\infty)$ " such that $f^{\prime}$ is not ultimately monotonic. In both the following examples the reader will check the asymptotic formulas for $f^{\prime \prime}$ showing that $f^{\prime \prime}$ changes sign infinitely often and that $f^{\prime}$ has no index of variation:

$$
\begin{gathered}
\left\{\begin{array}{l}
f_{5}(x):=x^{\alpha}+\sin \left(x^{\beta}\right), 0<\alpha / 2<\beta<\alpha, \\
f_{5}^{\prime \prime}(x)=x^{2(\beta-1)}\left[-\beta^{2} \sin \left(x^{\beta}\right)+o(1)\right] ;
\end{array}\right. \\
\left\{\begin{array}{l}
f_{6}(x):=x^{-\alpha}+x^{-\alpha-1} \sin \left(x^{\beta}\right), \alpha>0,1 / 2<\beta<1, \\
f_{6}^{\prime \prime}(x)=x^{2 \beta-\alpha-3}\left[-\beta^{2} \sin \left(x^{\beta}\right)+o(1)\right] .
\end{array}\right.
\end{gathered}
$$

(II) (Slow variation).

$$
f^{\prime}(x) / f(x)=o\left(x^{-1}\right) \Rightarrow f^{\prime}(x) \ll x^{-1} f(x) \ll x^{-1+\epsilon} \forall \epsilon>0 \Rightarrow f^{\prime}(x)=o(1),
$$

and $f^{\prime}$ may be ultimately monotonic, as for the functions in (2.9) or it may even change sign infinitely often as for the functions " $2+\sin \left[(\log x)^{\delta}\right]$ and $\exp \left(2+\sin \left[(\log x)^{\delta}\right]\right), 0<\delta<1 ”$.

(III) (Rapid variation).

$$
\begin{gathered}
x f^{\prime}(x) / f(x) \rightarrow+\infty \Rightarrow\left\{\begin{array}{l}
f^{\prime}(x)>0 \text { ultimately, } \\
f^{\prime}(x) \gg x^{\alpha}, x \rightarrow+\infty, \forall \alpha>0 ;
\end{array}\right. \\
x f^{\prime}(x) / f(x) \rightarrow-\infty \Rightarrow\left\{f^{\prime}(x)<0 \text { ultimately, } \int^{+\infty}\left|f^{\prime}\right|<+\infty\right\} .
\end{gathered}
$$

For $f \in \mathcal{R}_{-\infty}(+\infty)$ the additional condition " $f$ ' monotonic" grants that $f^{\prime}$ satisfies the same asymptotic estimates as $f$ :

$$
f^{\prime}(x) \ll x^{-\alpha}, x \rightarrow+\infty, \forall \alpha>0 .
$$

We do not know if relations in (2.102), or even the simple relation $f^{\prime}(+\infty)=0$, are satisfied by any $f \in \mathcal{R}_{-\infty}(+\infty)$.

Proof of part (III). The estimates for $f^{\prime}$ in (2.100) follow from

$$
x^{-\alpha} f^{\prime}(x)=\left(\frac{x f^{\prime}(x)}{f(x)}\right) \cdot\left(\frac{f(x)}{x^{\alpha+1}}\right)
$$

where both factors tend to $+\infty$ by (2.41). This simple argument does not work for $f \in \mathcal{R}_{-\infty}(+\infty)$ because the indeterminate form " $(-\infty) \cdot 0$ " appears on the left in (2.103). The summability of $f^{\prime}$ in (2.101) simply follows from $f(+\infty)=0$. The estimates in (2.102) follow from those in (2.41) by a classical result which requires either " $f$ everywhere differentiable and $f^{\prime}$ monotonic" or " $f \in A C[T,+\infty)$ and $f^{\prime}$ monotonic on $[T,+\infty) \backslash N$ with mis $N=0$ ": see [12] both for historical references in the introduction and for generalizations.

Notice that it is easy to give an example of a function $g \sim f$ such that $f \in \mathcal{R}_{-\infty}(+\infty)$ and $g^{\prime}(+\infty)$ does not exist in $\overline{\mathbb{R}}$ with $g^{\prime}$ either bounded or not:

$$
\left\{\begin{array}{l}
g(x):=\mathrm{e}^{-x}+\mathrm{e}^{-a x} \sin \left(\mathrm{e}^{b x}\right) \sim \mathrm{e}^{-x},(a>1, b \geq a), \\
g^{\prime}(x)=-\mathrm{e}^{-x}-a \mathrm{e}^{-a x} \sin \left(\mathrm{e}^{b x}\right)+b \mathrm{e}^{(b-a) x} \cos \left(\mathrm{e}^{b x}\right)=b \mathrm{e}^{(b-a) x} \cos \left(\mathrm{e}^{b x}\right)+o(1) ;
\end{array}\right.
$$


but in this case the limit " $\lim _{x \rightarrow+\infty} x g^{\prime}(x) / g(x)$ " does not exist as well.

As far as the possible index of variation of the first derivative is concerned notice that if $f \in \mathcal{R}(+\infty)$ then $\left|f^{\prime}\right|$ may have no index of variation at $+\infty$ as shown by the following counterexamples where the term "oscillatory" means that the pertinent function changes sign infinitely often as $x \rightarrow+\infty$ :

$$
\begin{cases}x^{\alpha}+\sin x \in \mathcal{R}_{\alpha}(+\infty), 1<\alpha<2, & \left(x f^{\prime \prime} / f^{\prime} \text { oscillatory and unbounded }\right) ; \\ x^{2}+\sin x \in \mathcal{R}_{\alpha}(+\infty), 1<\alpha \leq 2, & \left(x f^{\prime \prime} / f^{\prime} \text { oscillatory and bounded }\right) ; \\ 2+\sin \left[(\log x)^{\alpha}\right] \in \mathcal{R}_{0}(+\infty), 0<\alpha<1, & \left(f^{\prime} \text { oscillatory and } f^{\prime}(+\infty)=0\right) ; \\ 2 \mathrm{e}^{x}+\sin \left(\mathrm{e}^{x}\right) \in \mathcal{R}_{+\infty}(+\infty), & \left(x f^{\prime \prime} / f^{\prime}\right. \text { oscillatory and unbounded); } \\ \frac{1}{2} \mathrm{e}^{-x}+\mathrm{e}^{-2 x} \sin \left(\mathrm{e}^{x / 2}\right) \in \mathcal{R}_{-\infty}(+\infty), & \left(x f^{\prime \prime} / f^{\prime} \text { oscillatory and unbounded }\right) .\end{cases}
$$

But if $\left|f^{\prime}\right|$ has an index of variation then there are precise important links between the two indexes. The results in the next proposition are essential in the higher-order theory and to understand why restrictions on the indexes are sometimes required.

Proposition 2.6. (Index of variation of the first derivative). (I) If $f \in A C^{1}[T,+\infty$ ) and if both $f$ and $\left|f^{\prime}\right|$ have indexes of variation at $+\infty$, respectively $\alpha$ and $\alpha^{\prime}$, then:

$$
\left\{\begin{array}{l}
\alpha^{\prime}=\alpha-1 \text { if } \alpha \in \mathbb{R} \backslash\{0\} \text { or if } \alpha=0 \text { and } \lim _{x \rightarrow+\infty} f(x)=\text { either } 0 \text { or }+\infty ; \\
\alpha^{\prime}=\alpha \text { if } \alpha= \pm \infty .
\end{array}\right.
$$

In the case $\alpha=0$ and without the stated additional condition on $f(+\infty)$, it may happen that $\left|f^{\prime}\right| \in R_{\alpha^{\prime}}(+\infty)$ with $-\infty \leq \alpha^{\prime} \leq-1$ as shown by the simple examples.

$$
1+\mathrm{e}^{-x} ; 1+x^{-\delta}(\delta>0) ; 1+(\log x)^{-\delta}(\delta>0) ;
$$

but it cannot be $\alpha^{\prime}>-1$. Hence for $\alpha \in \mathbb{R}$ it always is: $\alpha^{\prime} \leq \alpha-1$.

(II) If $f \in \mathcal{R}_{\alpha}(+\infty),\left|f^{\prime}\right| \in \mathcal{R}_{\beta}(+\infty)$ for some $\alpha, \beta \in \mathbb{R}$ then

$$
f^{\prime \prime}(x)=\alpha \beta x^{-2} f(x)+o\left(x^{-2} f(x)\right), x \rightarrow+\infty .
$$

If $f \in \mathcal{R}_{\alpha}(+\infty)$ for some $\alpha \in \mathbb{R} \backslash\{0\}$ then $\left|f^{\prime}\right| \in \mathcal{R}_{\alpha-1}(+\infty)$ if and only if

$$
f^{\prime \prime}(x)=\gamma x^{-2} f(x)+o\left(x^{-2} f(x)\right), x \rightarrow+\infty ;
$$

for some $\gamma \in \mathbb{R}$ and in such a case it is necessarily $\gamma=\alpha(\alpha-1)$.

$$
\begin{gathered}
\text { (III) If either " } f,\left|f^{\prime}\right| \in \mathcal{R}_{+\infty}(+\infty) \text { " or " } f,\left|f^{\prime}\right| \in \mathcal{R}_{-\infty}(+\infty) \text { " then } \\
\lim _{x \rightarrow+\infty} x^{2} f^{\prime \prime}(x) / f(x)=+\infty ;
\end{gathered}
$$

and we do not know whether the partial converse holds true i.e. if both conditions “ $f \in \mathcal{R}_{ \pm \infty}(+\infty)$ and (2.110)" imply $\left|f^{\prime}\right| \in \mathcal{R}_{ \pm \infty}(+\infty)$.

Proof. Proof of part (I) is taken from ([13]; proof of Lemma 2.3, p. 111]). By hypothesis the following two limits exist in $\overline{\mathbb{R}}$ :

$$
\lim _{x \rightarrow+\infty} x f^{\prime}(x) / f(x) \equiv \alpha, \quad \lim _{x \rightarrow+\infty} x f^{\prime \prime}(x) / f^{\prime}(x) \equiv \alpha^{\prime} .
$$

We now evaluate $\alpha$ by L'Hospital's rule noticing that: " $0<\alpha \leq+\infty$ ” implies " $f(+\infty)=+\infty$ ", whereas “ $-\infty<\alpha<0$ " implies " $f(+\infty)=0$ " and the first limit in 
(2.111) implies " $\lim _{x \rightarrow+\infty} x f^{\prime}(x)=0$ ". In both cases the rule may be applied and

$$
\alpha=\lim _{x \rightarrow+\infty} \frac{f^{\prime}(x)+x f^{\prime \prime}(x)}{f^{\prime}(x)}=1+\alpha^{\prime} .
$$

The same argument is valid for $\alpha=0$ and the stated restriction on $f(+\infty)$. It remains the case " $\alpha=-\infty$ " which implies " $f(+\infty)=0$ " and this condition leads to excluding the following contingencies for the indicated reasons:

(i) $\alpha^{\prime}=+\infty$ would imply " $\left|f^{\prime}(+\infty)\right|=+\infty$ " hence " $f(+\infty)=+\infty$ (being $f>0$ )".

(ii) $-1<\alpha^{\prime}<+\infty$ would imply “ $x\left|f^{\prime}(x)\right| \in \mathcal{R}_{\alpha^{\prime}+1}(+\infty)$ " hence “ $\lim _{x \rightarrow+\infty} x\left|f^{\prime}(x)\right|=+\infty$ ”, and by L'Hospital:

$$
\lim _{x \rightarrow+\infty}\left|\left(\int_{T}^{x} f^{\prime}\right) / x f^{\prime}(x)\right|=\lim _{x \rightarrow+\infty}\left|\frac{f^{\prime}(x)}{f^{\prime}(x)+x f^{\prime \prime}(x)}\right|=\lim _{x \rightarrow+\infty} 1 /\left|1+\frac{x f^{\prime \prime}(x)}{f^{\prime}(x)}\right|=1 /\left|1+\alpha^{\prime}\right|,
$$

which is a positive real number; hence " $\int_{T}^{+\infty}\left|f^{\prime}\right|=+\infty$ " which would imply " $f(+\infty)=+\infty$ ".

(iii) $-\infty<\alpha^{\prime}<-1$ would imply “ $x\left|f^{\prime}(x)\right| \in \mathcal{R}_{\alpha^{\prime}+1}(+\infty)$ " hence “ $\lim _{x \rightarrow+\infty} x\left|f^{\prime}(x)\right|=0$ ", and this would imply the contradiction: $-\infty=\lim _{x \rightarrow+\infty} x f^{\prime}(x) / f(x) \stackrel{\text { as in }(2.112)}{=} 1+\alpha^{\prime} \in \mathbb{R}$.

(iv) The case $\alpha^{\prime}=-1$ must be treated in a different way using the estimates in (2.19) and (2.41). In our present proof we have $\alpha=-\infty$ and $\alpha^{\prime}=-1$, hence

$$
f(x) \ll x^{-\epsilon}, x \rightarrow+\infty, \forall \epsilon>0 ; x^{-1-\epsilon} \ll\left|f^{\prime}(x)\right| \ll x^{-1+\epsilon}, x \rightarrow+\infty, \forall \epsilon>0 ;
$$

and there are two a-priori contingencies concerning the integral $\int^{+\infty}\left|f^{\prime}\right|$. Its divergence would imply $f(+\infty)=+\infty$ which cannot be; in the other case we would have

$$
\int^{+\infty}\left|f^{\prime}\right|<+\infty \Rightarrow f(x)=-\int_{x}^{+\infty} f^{\prime}=\int_{x}^{+\infty}\left|f^{\prime}\right| \gg \int_{x}^{+\infty} t^{-1-\epsilon} \mathrm{d} t=\frac{x^{-\epsilon}}{\epsilon}, x \rightarrow+\infty,
$$

contradicting the first relation in (2.114). Notice that the procedure used to prove this last case works for any $\alpha^{\prime} \in \mathbb{R}$ as well.

The last assertion in the statement, namely "it cannot be $\alpha^{\prime}>-1$ ", follows from the calculations in the case (ii): $\alpha^{\prime}>-1$ would imply $f(+\infty)=+\infty$, and the claim in (2.106) concerning $\alpha=0$ would imply $\alpha^{\prime}=-1$ : a contradiction. The proof of part (I) is complete.

For part (II) the assumptions for (2.108) are:

$$
f^{\prime}(x)=\alpha x^{-1} f(x)+o\left(x^{-1} f(x)\right), f^{\prime \prime}(x)=\beta x^{-1} f^{\prime}(x)+o\left(x^{-1} f^{\prime}(x)\right), x \rightarrow+\infty,
$$

whence (2.108) follows. Viceversa assume $f \in \mathcal{R}_{\alpha}(+\infty)$ for some $\alpha \in \mathbb{R} \backslash\{0\}$ and relation (2.109). The restriction $\alpha \neq 0$ implies $f(x) \sim(1 / \alpha) x f^{\prime}(x)$ and (2.109) yields

$$
f^{\prime \prime}(x)=(\gamma / \alpha) x^{-1} f^{\prime}(x)+o\left(x^{-1} f^{\prime}(x)\right), x \rightarrow+\infty,
$$

which means that $\left|f^{\prime}\right| \in \mathcal{R}_{(\gamma / \alpha)}(+\infty)$ and the first claim in (2.106) implies $(\gamma / \alpha)=\alpha-1$.

Last, relation (2.110) follows from the decomposition 


$$
\frac{x^{2} f^{\prime \prime}}{f}=\left(\frac{x f^{\prime \prime}}{f^{\prime}}\right) \cdot\left(\frac{x f^{\prime}}{f}\right) \rightarrow+\infty,
$$

as the factors on the right diverge either both to $+\infty$ or both to $-\infty$.

\section{The Theory of Higher-Order Regular or Smooth Variation}

\subsection{The Concept of Higher-Order Regular Variation}

By the foregoing proposition we can define unambiguously some concepts of "higher-order asymptotic variation" separating the cases of regular variation (in this section) and rapid variation (in the next section).

Definition 3.1. (Regular variation of higher order). A function $f \in A C^{n-1}[T,+\infty), n \geq 1$, is termed "regularly varying at $+\infty$ (in the strong sense) of order $n$ " if each of the functions $|f|,\left|f^{\prime}\right|, \cdots,\left|f^{(n-1)}\right|$ never vanishes on a neighborhood of $+\infty$ and is regularly varying at $+\infty$ with its own index of variation according to Proposition 2.6. If this is the case we use notation

$$
f \in\left\{\mathcal{R}_{\alpha}(+\infty) \text { of order } n\right\}, \alpha:=\text { "the index of } f \text { " } \in \mathbb{R} \text {. }
$$

Whenever needed we denote the indexes of the derivatives as follows.

$$
\left|f^{(k)}\right| \in \mathcal{R}_{\alpha_{k}}(+\infty), 0 \leq k \leq n-1 ; \alpha_{0} \equiv \alpha .
$$

Remarks. 1. It is essential to consider the absolute values in order to not impose a-priori restrictions on the signs of the derivatives. Saying that " $f$ is regularly varying of order 1" means that " $|f|$ is regularly varying in the sense of Definition 2.1". The functions in (2.7) are regularly-varying at $+\infty$ (in the strong sense) of any order $n$. The index $\alpha_{1}$ was denoted by $\alpha^{\prime}$ in Proposition 2.6.

2. A nonzero constant belongs to the class $\left\{\mathcal{R}_{0}(+\infty)\right.$ of order 1$\}$ and no more because its derivative has no index of variation; a polynomial of exact algebraic degree $n \geq 1$ belongs to the class $\left\{\mathcal{R}_{n}(+\infty)\right.$ of order $\left.n+1\right\}$. Hence if a polynomial satisfies (3.1) for some $\alpha \in \mathbb{R}$ then $\alpha \geq n-1$.

3. If (3.1) holds true then, by Proposition 2.6-(I):

$$
\begin{gathered}
\alpha \notin\{0,1, \cdots, n-2\}, n \geq 2 \Rightarrow \alpha_{k}=\alpha-k, 1 \leq k \leq n-1 ; \\
\alpha \equiv k_{0} \in\{0,1, \cdots, n-2\}, n \geq 2 \Rightarrow \begin{cases}\alpha_{k}=k_{0}-k, & 0 \leq k \leq k_{0} ; \\
\alpha_{k_{0}+1}=\beta, & \text { for some } \beta \leq-1 ; \\
\alpha_{k}=\beta-\left(k-k_{0}+1\right), & k_{0}+1 \leq k \leq n-1 .\end{cases}
\end{gathered}
$$

By (2.106) the inference in (3.3) may well hold true without the stated restriction whenever " $\alpha_{p}=0$ " for some $p$ and " $f^{(p)}(+\infty)=$ either 0 or $+\infty$ ". In any case, though not all the indexex $\alpha_{k}$ may be uniquely determined a priori, there are precise and fundamental asymptotic relations linking each $f^{(k)}$ to $f$ for $1 \leq k \leq n$, and depending only on $\alpha$. Notice that, by our agreements, a notation like

" $f \in\left\{\mathcal{R}_{0}(+\infty)\right.$ of order $\left.n \geq 2\right\}$ " implies that the involved derivatives of $f$ are regularly varying; for instance it is misleading to write " $1+\mathrm{e}^{-x} \in\left\{\mathcal{R}_{0}(+\infty)\right.$ of order $\left.n \geq 2\right\}$ ": 
see examples in (3.40) below.

Proposition 3.1. (Principal parts of higher derivatives in case of regular variation).

(I) If $f \in\left\{\mathcal{R}_{\alpha}(+\infty)\right.$ of order $\left.n\right\}, n \geq 1$, then relations

$$
\begin{aligned}
& f^{(k)}(x) / f(x) \\
& =\alpha(\alpha-1) \cdots(\alpha-k+1) x^{-k}+o\left(x^{-k}\right) \equiv \alpha^{-k} x^{-k}+o\left(x^{-k}\right), x \rightarrow+\infty, 1 \leq k \leq n,
\end{aligned}
$$

hold true whichever $\alpha \in \mathbb{R}$ may be. For $\alpha \neq 0$ they may be written as

$$
f^{(k)}(x)=\frac{(\alpha-1) \cdots(\alpha-k+1)}{\alpha^{k-1}} \frac{\left(f^{\prime}(x)\right)^{k}}{(f(x))^{k-1}}+o\left(\frac{\left(f^{\prime}(x)\right)^{k}}{(f(x))^{k-1}}\right), x \rightarrow+\infty, 1 \leq k \leq n .
$$

(II) (Partial converse). If $f \in A C^{n-1}[T,+\infty), n \geq 2$, then $f \in\left\{\mathcal{R}_{\alpha}(+\infty)\right.$ of order $\left.n\right\}$ for some $\alpha \notin\{0,1, \cdots, n-2\}$ if and only if the following relations hold true

$$
f^{(k)}(x) / f(x)=\gamma_{k} x^{-k}+o\left(x^{-k}\right), x \rightarrow+\infty, 1 \leq k \leq n,
$$

with suitable constants $\gamma_{k}$ such that

$$
\gamma_{1}, \cdots, \gamma_{n-1} \neq 0 ;\left(\text { no restriction on } \gamma_{n}\right) \text {. }
$$

If this is the case then: $\gamma_{k}=\alpha^{\underline{k}}, 1 \leq k \leq n$.

Proof. (I) Both claims for $n=2$ are contained in Proposition 2.6-(I), (II): if $\alpha \neq 0$ in (2.108) then $\beta=\alpha-1$ and if $\alpha=0$ then obviously $\alpha \beta=\alpha(\alpha-1)$. For part (I) and $n \geq 3$ we have by assumption the set of relations

$$
\left\{\begin{array}{c}
f^{\prime}(x)=\alpha x^{-1} f(x)+o\left(x^{-1} f(x)\right) \\
f^{\prime \prime}(x)=\alpha_{1} x^{-1} f^{\prime}(x)+o\left(x^{-1} f^{\prime}(x)\right) \\
\vdots \\
f^{(k)}(x)=\alpha_{k-1} x^{-1} f^{(k-1)}(x)+o\left(x^{-1} f^{(k-1)}(x)\right)
\end{array}\right.
$$

Replacing the relation for $f^{(k-1)}(x)$ into the last relation we get

$$
f^{(k)}(x)=\alpha_{k-1} \alpha_{k-2} x^{-2} f^{(k-2)}(x)+o\left(x^{-2} f^{(k-2)}(x)\right)
$$

and iterating the procedure yields

$$
f^{(k)}(x) / f(x)=\alpha \alpha_{1} \cdots \alpha_{k-1} x^{-k}+o\left(x^{-k}\right), x \rightarrow+\infty, 1 \leq k \leq n,
$$

which by (2.106) coincides with (3.5) under the assumptions in (3.3). Under the assumptions in (3.4) we get relations in (3.5) for $1 \leq k \leq k_{0}$ and, being $\alpha_{k_{0}}=0$, relations

$$
f^{(k)}(x) / f(x)=o\left(x^{-k}\right), x \rightarrow+\infty, k_{0}+1 \leq k \leq n .
$$

In any case (3.5) hold true for $1 \leq k \leq n$. Relations in (3.6) simply follow from the inference

$$
f^{\prime}(x) \sim \alpha x^{-1} f(x) \Rightarrow x^{-k} f(x) \sim \alpha^{-k}\left(f^{\prime}(x)\right)^{k}(f(x))^{1-k} .
$$

For part (II) we must prove that relations (3.7)-(3.8) imply

$$
f^{(k)}(x) / f^{(k-1)}(x)=x^{-1}[\alpha-k+1+o(1)], x \rightarrow+\infty, 1 \leq k \leq n .
$$


The claim for $n=2$ is contained in Proposition 2.6-(II) and we proceed by induction assuming the claim true for a certain $k \leq n-1$. Supposing

$$
f^{(i)}(x) / f(x)=\gamma_{i} x^{-i}+o\left(x^{-i}\right), x \rightarrow+\infty, 1 \leq i \leq k+1 \text {, with } \gamma_{i} \text { satisfying (3.8), }
$$

the inductive hypothesis implies

$$
\gamma_{i}=\alpha^{i} \neq 0 \quad \text { and } \quad f^{(i)}(x) / f^{(i-1)}(x) \sim(\alpha-i+1) x^{-1} \quad \text { for } 1 \leq i \leq k ;
$$

and we must prove the relation in (3.7) with $k$ replaced by $k+1$. We express $f$ in terms of $f^{(k)}$ from the relation in (3.14) for $i=k$

$$
f(x) \sim\left(\alpha^{\underline{k}}\right)^{-1} x^{k} f^{(k)}(x),
$$

and replace this expression into the relation in (3.14) for $i=k+1$ so obtaining

$$
f^{(k+1)}(x) / f^{(k)}(x) f^{(k)}(x)=x^{-1}\left[\gamma_{k+1}\left(\alpha^{\underline{k}}\right)^{-1}+o(1)\right] \text {. }
$$

For $k=n-1$ this is the thesis without any restriction on $\gamma_{n}$; for $k<n-1$ the second relation in (3.15) implies $f^{(k-1)} \in \mathcal{R}_{\alpha-k+1}(+\infty)$ with $\alpha-k+1 \neq 0$ by assumption; it follows from (2.106) that $f^{(k)} \in \mathcal{R}_{\alpha-k}(+\infty)$. Hence in (3.17) it must be

$$
\gamma_{k+1}\left(\alpha^{\underline{k}}\right)^{-1}=\alpha-k \text { i.e. } \gamma_{k+1}=\alpha^{\frac{k+1}{1}}
$$

and the proof is over.

As noticed in the proof, (3.5) holds true for any $\alpha$, hence if the coefficient in the right-hand side vanishes for a certain value of $k$, say $\bar{k}$, it vanishes for all $k \geq \bar{k}$. The restriction $\alpha \notin\{0,1, \cdots, n-2\}$, used in the proof of part (II), cannot be suppressed otherwise any circumstance may occur. First counterexample:

$$
\left\{\begin{array}{l}
\left.\left.f(x):=2+\sin \left[(\log x)^{\delta}\right], x \in\right] 1,+\infty\right), 0<\delta<1 \\
f^{\prime}(x)=o\left(x^{-1} f(x)\right), f^{\prime \prime}(x)=o\left(x^{-2} f(x)\right)
\end{array}\right.
$$

wherein $f \in \mathcal{R}_{0}(+\infty), \alpha=0=n-2$, and $f^{\prime}$ cannot be regularly varying as it has alternating signs.

Second counterexample:

$$
f(x):=x^{n}+\mathrm{e}^{-x} \in\left\{\mathcal{R}_{n}(+\infty) \text { of order } n+1\right\} \text { (and no more), }
$$

though the relations in (3.7) hold true for each $k \geq 1$ : but $\gamma_{k}=0$ for $k \geq n+1$.

Third example:

$$
f(x):=1+\left\{\begin{array}{l}
(\log x)^{\delta}, \delta \neq 0, \\
x^{-\delta}, \delta>0
\end{array} \in\left\{\mathcal{R}_{0}(+\infty) \text { of any order } n \in \mathbb{N}\right\},\right.
$$

and relations in (3.7) hold true with $\gamma_{k}=0$ for each $k \geq 2$.

\subsection{The Concept of Higher-Order Smooth Variation}

The second counterexample above shows that the set of relations in (3.5) in themselves 
do not grant that all the involved derivatives be regularly varying: it may well occur an abrupt transition from regular variation to rapid variation at a certain order of derivation. This is the main motivation for our Definition 3.1. But the asymptotic relations for $f^{(k)} / f$ are most important in applications and in this subsection we report three characterizations of these relations encountered in the literature and valid for any $\alpha$ : the first deals with the derivatives of the ratio in (2.3), $x f^{\prime}(x) / f(x)$, and is used in the monograph by Lantsman [9]; the second is a slight variant dealing with the derivatives of the logarithmic derivative $f^{\prime}(x) / f(x)$; the third highlights the behavior at $+\infty$ of the derivatives of the associated function $\phi(x):=\log \left[f\left(\mathrm{e}^{x}\right)\right]$. This last characterization is a nontrivial and useful result proved by Balkema, Geluk and de Haan ([7]; Lemma 9, p. 410) using an ingenious device.

Proposition 3.2. (Several characterizations). For an $f \in A C^{n-1}[T,+\infty), n \geq 1$, $f(x)>0 \forall x$ large enough, the following four sets of asymptotic relations, for a fixed $\alpha \in \mathbb{R}$, are equivalent to each other.

$$
\begin{gathered}
x^{k} f^{(k)}(x) / f(x)=\alpha(\alpha-1) \cdots(\alpha-k+1)+o(1), x \rightarrow+\infty, 1 \leq k \leq n ; \\
\left\{\begin{array}{l}
x f^{\prime}(x) / f(x)=\alpha+o(1), x \rightarrow+\infty ; \\
\left(x f^{\prime}(x) / f(x)\right)^{(k)}=o\left(x^{-k}\right), x \rightarrow+\infty, 1 \leq k \leq n ;
\end{array}\right. \\
\left\{\begin{array}{l}
f^{\prime}(x) / f(x)=\alpha x^{-1}+o\left(x^{-1}\right), x \rightarrow+\infty ; \\
\left(f^{\prime}(x) / f(x)\right)^{(k)}=\alpha \cdot D^{k} x^{-1}+o\left(x^{-k-1}\right), x \rightarrow+\infty, 1 \leq k \leq n ;
\end{array}\right. \\
\left\{\begin{array}{l}
\phi(x):=\log \left[f\left(\mathrm{e}^{x}\right)\right] ; \phi^{\prime}(x)=\alpha+o(1), x \rightarrow+\infty ; \\
\phi^{(k)}(x)=o(1), x \rightarrow+\infty, 2 \leq k \leq n .
\end{array}\right.
\end{gathered}
$$

The reader will notice in the proof that the differential expressions " $x^{k} f^{(k)}(x) / f(x)$ " stem out from successive differentiations of $\exp [\phi(\log x)]$.

Proof. We use notation $D^{k}:=\mathrm{d}^{k} / \mathrm{d} x^{k}$. To prove "(3.22) $\Leftrightarrow(3.23)$ " we use the identity

$$
\left(x f^{\prime}(x) / f(x)\right)^{(k+1)}=x\left(f^{\prime}(x) / f(x)\right)^{(k+1)}+(k+1)\left(f^{\prime}(x) / f(x)\right)^{(k)},
$$

from which, putting $k=0$, the equivalence easily follows for $n=1$. By induction suppose the equivalence true for a certain $n \geq 2$; (3.22) true for $n+1$ imply the relations in (3.23) true for $1 \leq k \leq n$ whereas (3.25), for $k=n$, yields

$$
\begin{aligned}
x\left(f^{\prime}(x) / f(x)\right)^{(n+1)} & =\left(x f^{\prime}(x) / f(x)\right)^{(n+1)}-(n+1)\left(f^{\prime}(x) / f(x)\right)^{(n)} \\
& =o\left(x^{-n-1}\right)-(n+1) \alpha \cdot D^{n} x^{-1}+o\left(x^{-n-1}\right) \\
& =\alpha x \cdot D^{n+1} x^{-1}+o\left(x^{-n-1}\right)
\end{aligned}
$$

which implies the relation in (3.23) for $k=n+1$. Viceversa, if (3.23) are true for $n+1$ then the relations in (3.23) are true for $1 \leq k \leq n$ whereas, for $k=n+1$, we get from (3.25): 


$$
\left(x f^{\prime}(x) / f(x)\right)^{(n+1)}=\alpha\left[x \cdot D^{n+1} x^{-1}+(n+1) \cdot D^{n} x^{-1}\right]+o\left(x^{-n-1}\right)=o\left(x^{-n-1}\right),
$$

the sum in square brackets being $\equiv 0$. Let us now consider the obvious relations concerning the function $\phi$ defined in (3.24) and valid for all $x \geq \mathrm{T}>0$ :

$$
\phi(x)=\log \left[f\left(\mathrm{e}^{x}\right)\right] \Leftrightarrow f(x)=\exp [\phi(\log x)] \Rightarrow x f^{\prime}(x) / f(x)=\phi^{\prime}(\log x) .
$$

The equivalence $(3.22) \Leftrightarrow(3.24)$ is contained in the following:

$$
\left\{\begin{array} { l } 
{ \phi ^ { \prime } ( x ) = \alpha + o ( 1 ) , } \\
{ \phi ^ { ( k ) } ( x ) = o ( 1 ) , 2 \leq k \leq n , }
\end{array} \Leftrightarrow \left\{\begin{array}{l}
D \phi(\log x)=\alpha x^{-1}+o\left(x^{-1}\right), \\
D^{k} \phi(\log x)=\alpha \cdot D^{k-1} x^{-1}+o\left(x^{-k}\right), 2 \leq k \leq n .
\end{array}\right.\right.
$$

First, it is elementary to prove by induction the formula

$$
D^{k} \phi(\log x)=(-1)^{k-1}(k-1) ! x^{-k} \cdot \phi^{\prime}(\log x)+x^{-k} \sum_{i=2}^{k} c_{i} \cdot \phi^{(i)}(\log x), k \geq 1,
$$

wherein $\phi^{(i)}(\log x):=\mathrm{d}^{i} /\left.\mathrm{d} t^{i} \phi(t)\right|_{t=\log x}$, and the explicit expressions of the coefficients $c_{i}$ are not needed except for $c_{k}=1$. If the set of relations on the left in (3.29) holds true then (3.30) at once implies the validity of the set on the right whereas we proceed by induction to prove the converse inference. Let the set of relations on the right in (3.29) holds true with $k$ replaced by $k+1$, then the inductive assumption grants all relations on the left in (3.29). Now we consider (3.30) with $k$ replaced by $k+1$, and solve it with respect to $\phi^{(k+1)}$ so getting

$$
x^{-k-1} \phi^{(k+1)}(\log x)=D^{k+1} \phi(\log x)-(-1)^{k} k ! x^{-k-1}[\alpha+o(1)]+o\left(x^{-k-1}\right)=o\left(x^{-k-1}\right),
$$

having used the relation in (3.29) for $D^{k+1} \phi(\log x)$ and $\lim _{x \rightarrow+\infty} \phi^{\prime}(\log x)=\alpha$. The last and most difficult equivalence is "(3.21) $\Leftrightarrow$ (3.24)" a direct proof of which via Faà di Bruno's formula would involve cumbersome calculations. We report the original proof in a somewhat simplified form explicitly writing the arguments of the involved functions, avoiding the use of a change of variable, and with some additional passages to motivate the technical ideas of the proof. From representation $f(x)=\exp [\phi(\log x)]$, by direct routine differentiation and factoring out common factors, we get

$$
\left\{\begin{aligned}
f^{\prime}(x)= & x^{-1} f(x) \phi^{\prime}(\log x), \\
f^{\prime \prime}(x)= & x^{-2} f(x)\left[\phi^{\prime}(\log x)\left(\phi^{\prime}(\log x)-1\right)+\phi^{\prime \prime}(\log x)\right], \\
f^{\prime \prime \prime}(x)= & x^{-3} f(x)\left\{\phi^{\prime}(\log x)\left(\phi^{\prime}(\log x)-1\right)\left(\phi^{\prime}(\log x)-2\right)+\phi^{\prime \prime \prime}(\log x)\right. \\
& \left.+\phi^{\prime \prime}(\log x)\left[\left(\phi^{\prime}(\log x)-1\right)\left(\phi^{\prime}(\log x)-2\right)+\phi^{\prime}(\log x)\right]\right\},
\end{aligned}\right.
$$

wherein we have used the expression of $f^{\prime}$ to get the final expression of $f^{\prime \prime}$ and the expression of $f^{\prime \prime}$ to get the final expression of $f^{\prime \prime \prime}$. It is clear that further differentiations yield expressions for the operators

$$
F_{k}(x):=x^{k} f^{(k)}(x) / f(x), k \geq 1,
$$

and we shall prove the following representation:

$$
\begin{aligned}
& F_{k}(x) \\
& =\phi^{\prime}(\log x)\left(\phi^{\prime}(\log x)-1\right) \cdots\left(\phi^{\prime}(\log x)-k+1\right)+\phi^{(k)}(\log x)+N_{k}(\log x), 1 \leq k \leq n,
\end{aligned}
$$


where $N_{k}(x)$ is a polynomial in $\phi^{\prime}(x), \cdots, \phi^{(k-1)}(x)$ each term of which contains a factor $\phi^{(i)}$ with $i \geq 2$. This is true for $k=1,2,3$ and a simple proof by induction, provided by the authors of [7], is based on an equation linking $F_{k}, F_{k}^{\prime}, F_{k+1}$ simply obtained by differentiation of (3.33):

$$
\begin{aligned}
F_{k}^{\prime}(x) & =k x^{k-1} \frac{f^{(k)}(x)}{f(x)}+x^{k} \frac{f^{(k+1)}(x)}{f(x)}-x^{k} \frac{f^{(k)}(x) f^{\prime}(x)}{(f(x))^{2}} \\
& =x^{-1}\left[\left(k-F_{1}(x)\right) F_{k}(x)+F_{k+1}(x)\right] .
\end{aligned}
$$

If now (3.34) is assumed true for a certain $k$ then, differentiating both sides and using (3.35) in the left-hand side, we get

$$
\begin{aligned}
& x^{-1}\left[\left(k-F_{1}(x)\right) F_{k}(x)+F_{k+1}(x)\right] \\
& =x^{-1} \phi^{\prime \prime}(\log x)\left[\sum_{j=0}^{k-1}\left(\prod_{\substack{i=0 \\
i \neq j}}^{k-1}\left(\phi^{\prime}(\log x)-i\right)\right]+x^{-1} \phi^{(k+1)}(\log x)+x^{-1} N_{k}^{\prime}(\log x),\right.
\end{aligned}
$$

whence

$$
\begin{aligned}
& F_{k+1}(x)=\left(F_{1}(x)-k\right) F_{k}(x)+\phi^{(k+1)}(\log x) \\
&+\phi^{\prime \prime}(\log x)\left[\sum_{j=0}^{k-1}\left[\prod_{\substack{i=0 \\
i \neq j}}^{k-1}\left(\phi^{\prime}(\log x)-i\right)\right]+N_{k}^{\prime}(\log x)\right. \\
& \stackrel{\text { by (3.34) }}{=} \prod_{i=0}^{k}\left(\phi^{\prime}(\log x)-i\right)+\phi^{(k+1)}(\log x)+N_{k+1}(\log x),
\end{aligned}
$$

where we have put

$$
\begin{aligned}
N_{k+1}(\log x):= & \left(F_{1}(x)-k\right)\left[\phi^{(k)}(\log x)+N_{k}(\log x)\right] \\
& +\phi^{\prime \prime}(\log x)\left[\sum_{j=0}^{k-1}\left(\prod_{\substack{i=0 \\
i \neq j}}^{k-1}\left(\phi^{\prime}(\log x)-i\right)\right)\right]+N_{k}^{\prime}(\log x),
\end{aligned}
$$

the right-hand side being a polynomial in $\phi^{\prime}(x), \cdots, \phi^{k}(x)$ each term of which contains a factor $\phi^{(i)}$ with $i \geq 2$, and this proves (3.34) for $k+1$. Starting from (3.34) the proof by induction of the equivalence " $(3.21) \Leftrightarrow(3.24)$ " is quite trivial. $\square$

Balkema, Geluk and de Haan ([7]; p. 412) call "smoothly varying of exponent (三 index) $\alpha$ " a positive $C^{\infty}$-function $f$ defined on a neighborhood of $+\infty$ such that the associated function $\phi$ satisfies the relations in (3.24) for all $k \in \mathbb{N}$. In our context we give

Definition 3.2. (Smooth variation of higher order). A function $f \in A C^{n-1}[T,+\infty), n \geq 1, \quad f(x) \neq 0 \forall x$ large enough, is termed "smoothly varying at $+\infty$ of order $n$ and index $\alpha$ " if the four equivalent properties in Proposition 3.2, referred to $|f|$, are satisfied. We denote this class by: $\left\{S \mathcal{R}_{\alpha}(+\infty)\right.$ of order $\left.n\right\}$.

Notice that in our definition $f$ is allowed to be either $>0$ or $<0$, the essential point being that it ultimately assumes only one strict sign. From Proposition 3.1-(II) and the examples (3.18)-(3.20) we get the following inclusions: 
$\left\{\begin{array}{l}\left\{\mathcal{R}_{\alpha}(+\infty) \text { of order } n\right\}=\left\{S \mathcal{R}_{\alpha}(+\infty) \text { of order } n\right\} \text { if } n=1 \text { or }\{n \geq 2, \alpha \neq 0,1, \cdots, n-2\} ; \\ \left\{\mathcal{R}_{\alpha}(+\infty) \text { of order } n\right\} \varsubsetneqq\left\{S \mathcal{R}_{\alpha}(+\infty) \text { of order } n\right\} \text { otherwise; }\end{array}\right.$

the reason of the strict inclusion being that some derivatives of a smoothly-varying function may vanish or change sign infinitely often. Examples.

$\begin{cases}f(x):=\text { constant } \neq 0 & \in\left\{S \mathcal{R}_{0}(+\infty) \text { of order } n\right\} \backslash\left\{\mathcal{R}_{0}(+\infty) \text { of order } n\right\} \text { for each } n \geq 2 ; \\ f(x):=1+x^{-\delta}, \delta>0, & \in\left\{\mathcal{R}_{0}(+\infty) \text { of any order } n \geq 2\right\} ; \\ f(x):=1+\mathrm{e}^{-x} & \in\left\{S \mathcal{R}_{0}(+\infty) \text { of order } n\right\} \backslash\left\{\mathcal{R}_{0}(+\infty) \text { of order } n\right\} \text { for each } n \geq 2 ; \\ f(x):=\left\{\begin{array}{l}2+\sin \left[(\log x)^{\delta}\right] \\ \text { with } 0<\delta<1\end{array}\right. & \in\left\{S \mathcal{R}_{0}(+\infty) \text { of order } n\right\} \backslash\left\{\mathcal{R}_{0}(+\infty) \text { of order } n\right\} \text { for each } n \geq 2 .\end{cases}$

In the third example all derivatives $f^{(k)}, k \geq 2$, are rapidly varying whereas in the fourth example they have no index of variation at $+\infty$. Let us consider the further example of a function already exibited in (2.22):

$$
\left\{\begin{array}{l}
f_{\delta}(x):=\exp \left[(\log x)^{\delta} \cdot \sin \left((\log x)^{\delta}\right)\right], \delta>0 \\
\liminf _{x \rightarrow+\infty} f_{\delta}(x)=0, \limsup _{x \rightarrow+\infty} f_{\delta}(x)=+\infty
\end{array}\right.
$$

If as in (3.24) we associate to $f_{\delta}$ the function $\phi_{\delta}(x):=x^{\delta} \sin \left(x^{\delta}\right)$ it is easy to check the following:

$$
\left\{\begin{array}{l}
0<\delta<1 / 2 \Rightarrow f_{\delta} \in\left\{\mathcal{R}_{0}(+\infty) \text { of order } 1\right\} ; f_{\delta}^{\prime} \text { oscillatory; } f_{\delta}^{\prime}(x)=o(1), x \rightarrow+\infty ; \\
0<\delta<n /(n+1) \Rightarrow f_{\delta} \in\left\{\mathcal{S R}_{0}(+\infty) \text { of order } n\right\} .
\end{array}\right.
$$

In fact we have $\phi_{\delta}^{\prime}(x)=\delta x^{\delta-1} \sin \left(x^{\delta}\right)+\delta x^{2 \delta-1} \cos \left(x^{\delta}\right)$ from whence an elementary induction proves the representation

$$
\begin{aligned}
& \left\{\begin{array}{l}
\phi_{\delta}^{(k)}(x)=\sum_{i} c_{i, k} x^{\delta_{i, k}} \omega_{i, k}(x), \\
\max _{i} \delta_{i, k}=(k+1) \delta-k ; \omega_{i, k}(x) \text { standing for one of the functions } \sin \left(x^{\delta}\right), \cos \left(x^{\delta}\right) ;
\end{array}\right. \\
& \text { implying } \phi_{\delta}^{(k)}(x)=o(1) \text { for each } k, 1 \leq k \leq n, \text { if } \delta<n /(n+1) .
\end{aligned}
$$

The anomalies in these examples make the definition of smooth variation a bit unsatisfying from a theoretical viewpoint unlike the definition of higher-order regular variation; they also show that the possible more complete locution "smooth regular variation" would not be appropriate; however it turns out that relations in (3.21)-(3.24), regardless of $\alpha$, are the right ones required in various applications. In the next proposition it is asserted that the derivative of a smoothly-varying function may not be smoothly-varying only if the index is zero whereas antiderivatives are always smoothly varying with suitable indexes.

Proposition 3.3. (Derivatives and integrals of smoothly varying functions). (I)

$$
f \in\left\{\mathcal{S R}_{\alpha}(+\infty) \text { of order } n \geq 2\right\}, \alpha \neq 0, f^{\prime} \in\left\{\mathcal{S R}_{\alpha-1}(+\infty) \text { of order } n-1\right\} .
$$

(II) If $f \in\left\{\mathcal{S R}_{\alpha}(+\infty)\right.$ of order $\left.n \geq 1\right\}$ then 


$$
\left\{\begin{array}{l}
\int^{+\infty} f \text { convergent } \Rightarrow \int_{x}^{+\infty} f(t) \mathrm{d} t \in\left\{\mathcal{S} \mathcal{R}_{\alpha+1}(+\infty) \text { of order } n+1\right\} \\
\int^{+\infty} f \text { divergent } \Rightarrow \int_{T}^{x} f(t) \mathrm{d} t, \text { with } T \text { large enough, } \in\left\{\mathcal{S R}_{\alpha+1}(+\infty) \text { of order } n+1\right\} .
\end{array}\right.
$$

The very same inferences in the case of regular variation, i.e. with $\mathcal{S R}$ replaced by $\mathcal{R}$, are included in Propositions 2.4-(I), 2.6-(I) and Definition 3.1.

Proof. We report the more elementary arguments used in ([8]; Prop. 1.8.1, p. 44) in preference to those in ([7]; Lemmas 10-11, p. 412). For (3.44) put $F:=f^{\prime}$ and notice that $f^{\prime}$ is ultimately of one strict sign as $f^{\prime}(x) \sim \alpha x^{-1} f(x)$; hence for each $k \in\{1, \cdots, n-1\}$ we may write by (3.21):

$$
\begin{aligned}
\frac{x^{k} F^{(k)}(x)}{F(x)} & \equiv \frac{x^{k+1} f^{(k+1)}(x)}{f(x)} \cdot \frac{f(x)}{x f^{\prime}(x)} \\
& =\frac{\alpha^{\frac{k+1}{\alpha}}}{\alpha}+o(1)=(\alpha-1) \cdots(\alpha-k)+o(1)=(\alpha-1)^{\underline{k}}+o(1) .
\end{aligned}
$$

To prove the first inference in (3.45) put $F(x):=\int_{x}^{+\infty} f(t) \mathrm{d} t$. Recalling that $f$ has ultimately one strict sign and that " $|f| \in \mathcal{R}_{\alpha}(+\infty)$ " Proposition 2.4-(I) implies $x F^{\prime}(x) / F(x)=|\alpha+1|+o(1)$ and by (3.21) we have for each $k \in\{1, \cdots, n+1\}$ :

$$
\begin{aligned}
\frac{x^{k} F^{(k)}(x)}{F(x)} & =\frac{x F^{\prime}(x)}{F(x)} \cdot \frac{x^{k-1} f^{(k-1)}(x)}{-f(x)} \\
& =-|\alpha+1| \alpha(\alpha-1) \cdots(\alpha-k+2)+o(1) \\
& = \begin{cases}o(1), & \text { if } \alpha=-1, \\
(\alpha+1) \alpha \cdots(\alpha-k+2)+o(1), & \text { if } \alpha<-1 .\end{cases}
\end{aligned}
$$

A similar proof in case of divergence. $\square$

Notice that, with $\phi$ defined in (3.24), $f$ is recovered by the formula $f(x)=\exp [\phi(\log x)]$ and that regular variation of $\phi$ may have ambiguous effects on $f$. The reader may check that:

$$
\phi \in \mathcal{R}_{\alpha}(+\infty) \Rightarrow \begin{cases}f \in \mathcal{R}_{+\infty}(+\infty) & \text { if } \alpha>1, \\ f \in \mathcal{R}_{0}(+\infty) & \text { if } \alpha<1,\end{cases}
$$

using “ $x f^{\prime}(x) / f(x)=\phi^{\prime}(\log x) \sim \alpha(\log x)^{-1} \phi(\log x)$ ” and the estimates in (2.19) referred to $\phi$. The case $\alpha=1$ remains undecided as shown by:

$$
\begin{aligned}
& \left\{\begin{array}{l}
\phi(x):=x+\delta \log x, \delta \in \mathbb{R} ; x f^{\prime}(x) / f(x) \sim\left(\ell_{2}(x)\right)^{\delta} ; \\
\text { hence }: \phi, f \in \mathcal{R}_{1}(+\infty) ;
\end{array}\right. \\
& \left\{\begin{array}{l}
\phi(x):=x(\log x)^{\delta}, \delta \neq 0 ; x f^{\prime}(x) / f(x) \sim\left(\ell_{2}(x)\right)^{\delta} ; \\
\text { hence }: \phi \in \mathcal{R}_{1}(+\infty), f \in \mathcal{R}_{+\infty}(+\infty) \text { if } \delta>0, f \in \mathcal{R}_{0}(+\infty) \text { if } \delta<0 .
\end{array}\right.
\end{aligned}
$$

To end this section let us ask ourselves what can be said about relations in (3.21) holding true with some unknown coefficients $\gamma_{k}$ on the right and we give a resultneeded in the sequel-concerning the circumstance $\gamma_{1}=\alpha \neq 0$.

Proposition 3.4. Let a function $f \in A C^{n-1}[T,+\infty), n \geq 2, f(x)>0 \forall x$ large 
enough, satisfy the following asymptotic relations:

$$
\begin{gathered}
x f^{\prime}(x) / f(x) \sim \alpha, x \rightarrow+\infty,(\alpha \neq 0) ; \\
x^{k} f^{(k)}(x) / f(x)=\gamma_{k}+o(1), 2 \leq k \leq n, x \rightarrow+\infty,
\end{gathered}
$$

for some unspecified constants $\gamma_{k}$. Then $f \in\left\{S \mathcal{R}_{\alpha}(+\infty)\right.$ of order $\left.n\right\}$

Proof. The claim amounts to state that the $\gamma_{k}$ 's coincide with the coefficients in (3.21). Now, if " $\gamma_{2}, \cdots, \gamma_{n-1} \neq 0$ " then Proposition 3.1-(II) states the stronger assertion " $f \in\left\{\mathcal{R}_{\alpha}(+\infty)\right.$ of order $\left.n\right\}$ " and, moreover, for $n=2$ there is nothing further to be proved. On the contrary if for $n \geq 3$ some coefficient $\gamma_{k}$ is zero then our claim will be proved once we show that all the successive coefficients are zero as well. In fact if “ $\gamma_{2}, \cdots, \gamma_{h} \neq 0$ " and " $\gamma_{h+1}=0$ " for some $h \leq n-2$, then:

$$
\begin{gathered}
x^{h} f^{(h)}(x) / f(x) \sim \gamma_{h}, x \rightarrow+\infty ; \quad x^{h+1} f^{(h+1)}(x) / f(x)=o(1), x \rightarrow+\infty ; \\
0=\lim _{x \rightarrow+\infty} \frac{x^{h+1} f^{(h+1)}(x)}{f(x)} \stackrel{\text { ‥ }}{=} \lim _{x \rightarrow+\infty} \frac{(h+1) x^{h} f^{(h+1)}(x)+x^{h+1} f^{(h+2)}(x)}{f^{\prime}(x)}=\cdots
\end{gathered}
$$

using (3.51) and then the secod relation in (3.53)

$$
\cdots=\lim _{x \rightarrow+\infty} \frac{(h+1) x^{h+1} f^{(h+1)}(x)}{\alpha f(x)}+\lim _{x \rightarrow+\infty} \frac{x^{(h+2)} f^{(h+2)}(x)}{\alpha f(x)}=\gamma_{h+2} / \alpha ;
$$

hence $\gamma_{h+2}=0$ and the proof is over. Note in passing that the second circumstance implies " $f^{(h)} \in \mathcal{R}_{0}(+\infty)$ " as the two relations in (3.53) imply:

$$
f^{(h+1)}(x) / f^{(h)}(x) \equiv\left(f^{(h+1)}(x) / f(x)\right) \cdot\left(f(x) / f^{(h)}(x)\right)=o\left(x^{-1}\right) .
$$

\section{The Theory of Higher-Order Rapid Variation}

Before giving the proper definition of higher-order rapid variation it is good to add some remarks about the additional condition

$$
\left(f(x) / f^{\prime}(x)\right)=o(1), x \rightarrow+\infty,
$$

appearing in Proposition 2.4-(II). The counterexample in (2.89) shows that this supplementary condition is almost necessary to obtain a meaningful general result about the asymptotic behavior of the antiderivatives of a rapidly-varying function. Now if in (2.84)-(2.85) we put $F(x):=\int_{T_{0}}^{x} f$, with the proper choice of $T_{0} \leq+\infty$, then the asymptotic behavior of $F$ can be reread as

$$
F(x) \sim\left(F^{\prime}(x)\right)^{2} / F^{\prime \prime}(x), x \rightarrow+\infty ;
$$

and, changing again notation, we have one of the following two equivalent relations:

$$
\begin{aligned}
& f^{\prime \prime}(x) \sim\left(f^{\prime}(x)\right)^{2} / f(x) ; \\
& f^{\prime \prime}(x) / f^{\prime}(x) \sim f^{\prime}(x) / f(x),\left(\text { i.e. } D_{\ell}\left(f^{\prime}(x)\right) \sim D_{\ell}(f(x))\right), x \rightarrow+\infty .
\end{aligned}
$$

For some applications conditions like those in (4.3) are necessary for meaningful 
general results as, e.g., in determining asymptotic expansions of antiderivatives and in another class of expansions studied in Part II, $\$ 11$, of this work: this justifies the following restricted concept of rapid variation.

Definition 4.1. (Rapid variation of higher order).

(I) (First order). A function $f \in A C^{1}[T,+\infty$ ), is called "rapidly varying at $+\infty$ (of order 1 ) in the strong restricted sense" if

$$
\begin{cases}f(x), f^{\prime}(x) \neq 0 & \forall x \text { large enough; } \\ f(x) / f^{\prime}(x)=o(x), & x \rightarrow+\infty \\ \left(f(x) / f^{\prime}(x)\right)^{\prime}=o(1), & x \rightarrow+\infty\end{cases}
$$

(II) (Higher order). A function $f \in A C^{n}[T,+\infty)$, is called "rapidly varying at $+\infty$ of order $n \geq 2$ in the strong restricted sense" if all the functions $f, f^{\prime}, \cdots, f^{(n-1)}$ are rapidly varying at $+\infty$ in the strong restricted sense and this amounts to say that the following conditions hold true as $x \rightarrow+\infty$ :

$$
\begin{gathered}
f^{(k)}(x) \neq 0 \quad \forall x \text { large enough and } 0 \leq k \leq n ; \\
f(x) / f^{\prime}(x)=o(x) ; f^{\prime}(x) / f^{\prime \prime}(x)=o(x) ; \cdots ; f^{(n-1)}(x) / f^{(n)}(x)=o(x) ; \\
\left(f(x) / f^{\prime}(x)\right)^{\prime}=o(1) ;\left(f^{\prime}(x) / f^{\prime \prime}(x)\right)^{\prime}=o(1) ; \cdots ;\left(f^{(n-1)}(x) / f^{(n)}(x)\right)^{\prime}=o(1) .
\end{gathered}
$$

If $f$ is rapidly varying at $+\infty$ of order $n \geq 2$ in the previous strong restricted sense then, by (2.106), all the functions $f, f^{\prime}, \cdots, f^{(n-1)}$ belong to the same class, either $\mathcal{R}_{-\infty}(+\infty)$ or $\mathcal{R}_{+\infty}(+\infty)$, hence we shall use notation $f \in\left\{\mathcal{R}_{ \pm \infty}(+\infty)\right.$ of order $\left.n\right\}$ to denote that $f$ enjoys the properties in (4.5)-(4.6)-(4.7) plus the corresponding value of the limit in (2.2). In most cases we shall not be interested in functions satisfying (4.5)-(4.6) but not (4.7), and so we use no additional notation to highlight the "strong restricted sense".

Remarks. 1. According to our definitions when we speak of a function $f$ rapidly varying (without specifying the order) we are using Definition 2.1 meaning that: $f \in A C[T,+\infty), f>0$ for $x$ large enough and (2.2) holds true. But when we speak of a function $f$ rapidly varying of order 1 (usually omitting the additional locution "in the strong restricted sense") we are using the stronger Definition 4.1 .

2. Conditions in (4.7) obviously imply those in (4.6) whereas, viceversa, complicated calculations in the attempt of proving (4.7) in addition to (4.6) may be usually saved using the classical result (already mentioned in the proof of Proposition 2.5) that: “ $\left\{g(x)=o(x), x \rightarrow+\infty ; g^{\prime}\right.$ monotonic $\} \Rightarrow g(x)=o(1), x \rightarrow+\infty$ ". For instance (4.5)(4.7) are trivially satisfied for the functions listed in (2.8) which are the most common functions rapidly varying at $+\infty$ of any order $n \in \mathbb{N}$ in our strong restricted sense.

As concerns the analogue of Proposition 3.1 it happens that relations in (3.5) have no analogues for rapidly-varying functions of higher order whereas those in (3.6) have so yielding a useful characterization of this class of functions.

Proposition 4.1. (Principal parts of higher derivatives in case of rapid variation). Let 
$f \in A C^{n}[T,+\infty)$ and conditions in (4.5) be satisfied; then

$f \in\left\{\mathcal{R}_{+\infty}(+\infty)\right.$ of order $\left.n\right\} \cup\left\{\mathcal{R}_{-\infty}(+\infty)\right.$ of order $\left.n\right\}$, i.e. conditions in (4.7) hold true, if and only if the following four equivalent sets of conditions are satisfied:

$$
\begin{gathered}
\left\{\begin{array}{c}
f^{\prime}(x) / f(x) \sim f^{\prime \prime}(x) / f^{\prime}(x) \sim \cdots \sim f^{(n)}(x) / f^{(n-1)}(x) \sim f^{(n+1)}(x) / f^{(n)}(x), \\
\text { i.e. } D_{\ell}\left(f^{(k)}(x)\right) \sim D_{\ell}(f(x)), x \rightarrow+\infty, 1 \leq k \leq n ;
\end{array}\right. \\
f^{(k+2)}(x) \sim\left(f^{(k+1)}(x)\right)^{2} / f^{(k)}(x), x \rightarrow+\infty, 0 \leq k \leq n-1 ; \\
f^{(k+2)}(x) / f(x) \sim\left(f^{\prime}(x) / f(x)\right)^{k+2} \equiv\left(D_{\ell}(f(x))\right)^{k+2}, x \rightarrow+\infty, 0 \leq k \leq n-1 ; \\
f(x) \equiv f^{\prime}(x) / D_{\ell}(f(x)) \sim f^{\prime \prime}(x) /\left(D_{\ell}(f(x))\right)^{2} \\
\sim \cdots \sim f^{(n+1)}(x) /\left(D_{\ell}(f(x))\right)^{n+1}, x \rightarrow+\infty .
\end{gathered}
$$

It follows that even $f^{(n+1)}(x) \neq 0$ for almost all $x$ large enough. Relations in (4.10) are formally obtained from those in (3.6) as the index $\alpha$ tends to $+\infty$. Relations in (4.10) imply the following asymptotic scale:

$$
f^{(n+1)}(x) \gg f^{(n)}(x) \gg \cdots \gg f^{\prime}(x) \gg f(x), x \rightarrow+\infty,
$$

whereas a different way of writing relations in (4.6) would give the weaker scale:

$$
f^{(n)}(x) \gg x^{-1} f^{(n-1)}(x) \gg x^{-2} f^{(n-2)}(x) \gg \cdots \gg x^{-(n-1)} f^{\prime}(x) \gg x^{-n} f(x), x \rightarrow+\infty .
$$

Proof. Relations in (4.9) and in (4.11) simply are different ways of rewriting relations respectively in (4.8) and in (4.10). Now inspecting (4.7) we have for $n=1$ :

$$
\left(f / f^{\prime}\right)^{\prime} \equiv 1-f f^{\prime \prime}\left(f^{\prime}\right)^{-2}=o(1) \Leftarrow f^{\prime \prime}\left(f^{\prime}\right)^{-2} \sim 1,
$$

which is (4.9) and (4.10) for $k=0$. Moreover, for any $k \in\{0,1, \cdots, n-1\}$ we have

$$
\begin{aligned}
& \left(f^{(k)} / f^{(k+1)}\right)^{\prime} \equiv 1-f^{(k)} f^{(k+2)}\left(f^{(k+1)}\right)^{-2}=o(1) \\
& \Leftrightarrow f^{(k)} f^{(k+2)}\left(f^{(k+1)}\right)^{-2} \sim 1 \Leftrightarrow f^{(k+2)} \sim\left(f^{(k+1)}\right)^{2} / f^{(k)} ;
\end{aligned}
$$

hence (4.7) are equivalent to (4.9). It remains to prove the equivalence between (4.9) and (4.10) for $n \geq 2$ and $k \geq 1$. Supposing relations in (4.9) true we start from the asymptotic relation involving $f^{(k+2)}$ and replace $f^{(k+1)}$ in the right-hand side with the analogous relation while leaving unaltered $f^{(k)}$; so we get

$$
f^{(k+2)} \sim\left(f^{(k+1)}\right)^{2} / f^{(k)} \sim\left(\left(f^{(k)}\right)^{2} /\left(f^{(k-1)}\right)\right)^{2} / f^{(k)}=\left(f^{(k)}\right)^{3} /\left(f^{(k-1)}\right)^{2} \sim \cdots
$$

If $k=1$ this gives $f^{(3)} \sim\left(f^{\prime}\right)^{3} / f^{2}$ which is (4.10) for $k=1$. If $k \geq 2$ we reapply the procedure to the last expression in (4.16) so getting

$$
f^{(k+2)} \sim\left(\left(f^{(k-1)}\right)^{2} / f^{(k-2)}\right)^{3} /\left(f^{(k-1)}\right)^{2}=\left(f^{(k-1)}\right)^{4} /\left(f^{(k-2)}\right)^{3} \sim \cdots
$$

If $k=2$ this gives $f^{(4)} \sim\left(f^{\prime}\right)^{4} / f^{3}$ which is (4.10) for $k=2$; and if $k \geq 3$ we repeat the procedure and get all relations in (4.10). Viceversa suppose (4.10) true and 
assume, by induction, that they imply (4.9), i.e. (4.8), for $k$ ranging in $\{0,1, \cdots, h\}$ for some $h \geq 1$, as this is trivially true for $h=0$. Let us write down the relations in (4.10) for $k=h, h+1$ :

$$
\begin{gathered}
f^{(h+2)} \sim\left(f^{\prime}\right)^{h+2} /(f)^{h+1} \\
f^{(h+3)} \sim\left(f^{\prime}\right)^{h+3} /(f)^{h+2}=\left(\left(f^{\prime}\right)^{h+2} /(f)^{h+1}\right) \cdot\left(f^{\prime} / f\right) .
\end{gathered}
$$

Using (4.18) and the relation in (4.8) involving the ratio $f^{(h+2)} / f^{(h+1)}$, which is true by the inductive hypothesis, we get

$$
f^{(h+3)} \sim f^{(h+2)} \cdot\left(f^{(h+2)} / f^{(h+1)}\right)=\left(f^{(h+2)}\right)^{2} / f^{(h+1)},
$$

which is the relation in (4.9) for $k=h+1$ and the proof is over.

An instructive counterexample concerning Definition 4.1 and the associated function. Let us consider the following function

$$
\left\{\begin{array}{l}
f_{c}(x):=c \mathrm{e}^{x}+\sin \left(\mathrm{e}^{x / 2}\right)=c \mathrm{e}^{x}+O(1), x \rightarrow+\infty ; c \neq 0 ; \\
f_{c}^{\prime}(x)=c \mathrm{e}^{x}+\frac{1}{2} \mathrm{e}^{x / 2} \cos \left(\mathrm{e}^{x / 2}\right) \sim c \mathrm{e}^{x} ; \\
f_{c}^{\prime \prime}(x)=c \mathrm{e}^{x}\left[1-\frac{1}{4 c} \sin \left(\mathrm{e}^{x / 2}\right)+o(1)\right] \equiv \begin{cases}c \cdot b(x) \mathrm{e}^{x} & \text { if }|c|>1 / 4, \\
c \cdot \omega(x) \mathrm{e}^{x} & \text { if }|c| \leq 1 / 4,\end{cases}
\end{array}\right.
$$

wherein ultimately " $0<M_{1} \leq b(x) \leq M_{2}<+\infty$ ", whereas $\omega(x)$ changes sign infinitely often though being bounded. The relations hold true:

$$
\left\{\begin{array}{l}
f_{c}(x) \sim f_{c}^{\prime}(x) \sim\left(f_{c}^{\prime}(x)\right)^{2} / f_{c}(x) \\
f_{c}^{\prime \prime}(x) / f_{c}^{\prime}(x) \sim b(x) \text { if }|c|>1 / 4 ; \quad f_{c}^{\prime \prime}(x) / f_{c}^{\prime}(x) \sim \omega(x) \text { if }|c| \leq 1 / 4
\end{array}\right.
$$

whence we infer that

$$
\left\{\begin{array}{l}
|c|>1 / 4 \Rightarrow f_{c}, f_{c}^{\prime} \in \mathcal{R}_{+\infty}(+\infty) ; f_{c}^{\prime \prime}(x) / f_{c}^{\prime}(x) \nsucc f_{c}^{\prime}(x) / f_{c}(x) ; \\
0<|c| \leq 1 / 4 \Rightarrow f_{c} \in \mathcal{R}_{+\infty}(+\infty), f_{c}^{\prime} \notin \mathcal{R}_{+\infty}(+\infty)
\end{array}\right.
$$

and condition (4.1) is not satisfied as

$$
\begin{aligned}
\left(f_{c} / f_{c}^{\prime}\right)^{\prime} & \equiv 1-f_{c} f_{c}^{\prime \prime}\left(f_{c}^{\prime}\right)^{-2}=1-c \mathrm{e}^{x} c \cdot\left\{\begin{array}{l}
b(x) \\
\omega(x)
\end{array}\right\} \cdot \mathrm{e}^{x} \cdot c^{-2} \mathrm{e}^{-2 x} \cdot(1+o(1)) \\
& =\left\{\begin{array}{l}
1-b(x)+o(1) \text { if }|c|>1 / 4 \\
1-\omega(x)+o(1) \text { if } 0<|c| \leq 1 / 4,
\end{array}\right.
\end{aligned}
$$

and $\lim _{x \rightarrow+\infty}\left(f_{c}(x) / f_{c}^{\prime}(x)\right)^{\prime}$ does not exist. So for any $c \neq 0$ we have an example of a function $f$ such that:

$$
f(x) / f^{\prime}(x) \sim 1 ; \lim _{x \rightarrow+\infty}\left(f(x) / f^{\prime}(x)\right)^{\prime} \text { does not exist; }
$$

though for $|c|>1 / 4$ the additional property " $\left(f / f^{\prime}\right)^{\prime}$ bounded" is satisfied.

For $|c|>1 / 4$ we also have an example of a function $f$ such that: 


$$
\left\{\begin{array}{l}
f, f^{\prime} \in \mathcal{R}_{+\infty}(+\infty) \text { i.e. } \lim _{x \rightarrow+\infty} x f^{\prime}(x) / f(x)=\lim _{x \rightarrow+\infty} x f^{\prime \prime}(x) / f^{\prime}(x)=+\infty, \\
f \notin\left\{\mathcal{R}_{+\infty}(+\infty) \text { of order } 1\right\} \text { in the strong restricted sense, } \\
\left(f / f^{\prime}\right)^{\prime} \text { bounded (but not convergent) at }+\infty .
\end{array}\right.
$$

As concerns the associated function in (3.24), $\phi_{c}(x):=\log \left[f_{c}\left(\mathrm{e}^{x}\right)\right]$, the second formula in (3.32) yields

$$
\phi_{c}^{\prime \prime}(\log x)=\frac{x^{2} f_{c}^{\prime \prime}(x)}{f_{c}(x)}-\frac{x f_{c}^{\prime}(x)}{f_{c}(x)}\left(\frac{x f_{c}^{\prime}(x)}{f_{c}(x)}-1\right)=x^{2} \cdot\left\{\begin{array}{c}
b(x)-1 \\
\omega(x)-1
\end{array}\right\}+x+o(x), x \rightarrow+\infty .
$$

Hence for any $c \neq 0$ we have an example of a function $f \in \mathcal{R}_{+\infty}(+\infty)$ satisfying the special additional relation $f(x) \sim f^{\prime}(x)$, and also $f^{\prime} \in \mathcal{R}_{+\infty}(+\infty)$ for $|c|>1 / 4$, such that the associated function $\phi$ has a second derivative oscillatory and unbounded. This shows that the properties of the associated function have little meaning, if any, in the context of rapid variation.

$A$ remark about the ratios $f^{(h)} / f^{(k)}$. In Definition 4.1-(II) relations in (4.6) imply the following chain:

$$
\begin{gathered}
f^{(k)}(x) \ll x f^{(k+1)}(x) \ll x^{2} f^{(k+2)}(x) \ll \cdots \ll x^{h} f^{(k+h)}(x), \\
x \rightarrow+\infty, 0 \leq k<k+h \leq n .
\end{gathered}
$$

A remark about $\lim _{x \rightarrow+\infty}\left(f / f^{\prime}\right)^{\prime}$. If a function $f \in A C^{1}[T,+\infty)$ with $f(x), f^{\prime}(x)$ ultimately $\neq 0$, satisfies condition

$$
\left(f(x) / f^{\prime}(x)\right)^{\prime}=a+o(1), x \rightarrow+\infty, \text { with } a \neq 0,
$$

an integration yields

$$
f(x) / f^{\prime}(x) \sim a x \text { i.e. } x f(x) / f^{\prime}(x) \sim 1 / a \text { i.e. } f \in \mathcal{R}_{1 / a}(+\infty) .
$$

Moreover the identity in (4.14) gives

$$
\begin{aligned}
& \qquad \frac{f(x) f^{\prime \prime}(x)}{\left(f^{\prime}(x)\right)^{2}}=(1-a)+o(1) \\
& \text { whence } \frac{f^{\prime \prime}(x)}{f^{\prime}(x)}=\frac{f^{\prime}(x)}{f(x)}[(1-a)+o(1)]=x^{-1}\left[\frac{(1-a)}{a}+o(1)\right],
\end{aligned}
$$

i.e. $f^{\prime} \in \mathcal{R}_{b}(+\infty)$ with $b=(1 / a)-1$ according to Proposition 2.6. In conclusion (4.29) implies " $f \in\left\{\mathcal{R}_{1 / a}(+\infty)\right.$ of order 2$\}$ ". Thus, if the asymptotic relation in (4.29) holds true for some real number $a$ and if it is known that $f \in \mathcal{R}_{ \pm \infty}(+\infty)$, then it must be $a=0$. Analogously, using the identity in (4.15) and under the regularity assumptions in Proposition 4.1, we prove that if

$$
f^{(k)}(x) f^{(k+2)}(x) / f^{(k+1)}(x)=c_{k}+o(1) \text { for some } k \in\{0,1, \cdots, n-1\},
$$

then

$$
\left(f^{(k)}(x) / f^{(k+1)}(x)\right)^{\prime}=\left(1-c_{k}\right)+o(1),
$$

and 


$$
f^{(k)}(x) / f^{(k+1)}(x)=\left(1-c_{k}\right) x+o(x),
$$

i.e. $f^{(k)} \in \mathcal{R}_{b}(+\infty)$ with $b=1 /\left(1-c_{k}\right)(+\infty)$. Hence if it is known a priori that $f^{(h)} \in \mathcal{R}_{ \pm \infty}(+\infty)$ for some $h \leq k$ then $c_{k}=1$. This fact will be needed in the sequel.

Corollary 4.2. (Summing up the behaviors of the higher derivatives). Let $f \in\left\{\mathcal{R}_{\alpha}(+\infty)\right.$ of any order $\left.n\right\}, \alpha \in \overline{\mathbb{R}}$. Then, as $x \rightarrow+\infty$ :

$$
\begin{gathered}
\alpha= \pm \infty \Rightarrow f^{(n)}(x) \sim\left(f^{\prime}(x) / f(x)\right)^{n} \cdot f(x), n \geq 2 ; \\
\alpha=0 \text { and } f^{\prime} \in \mathcal{R}_{-1}(+\infty) \Rightarrow f^{(n)}(x) \sim(-1)^{n-1}(n-1) ! x^{1-n} f^{\prime}(x), n \geq 2 ; \\
\alpha \in \mathbb{R} \Rightarrow f^{(n)}(x)=O\left(x^{-n} f(x)\right), n \geq 1 ; \\
\alpha \in \mathbb{R} \backslash \mathbb{N} \Rightarrow f^{(n)}(x) \sim \alpha^{\underline{n}} x^{-n} f(x), n \geq 1 .
\end{gathered}
$$

Examples.

$$
\begin{aligned}
& D^{n}\left(\mathrm{e}^{c x^{\gamma}}\right) \sim(c \gamma)^{n} x^{n(\gamma-1)} \mathrm{e}^{c x^{\gamma}}, x \rightarrow+\infty ; n \geq 1 ; c, \gamma \neq 0 ; \\
& D^{n}\left(\exp \left[c_{1} \exp \left(c_{2} x^{\gamma}\right)\right]\right) \sim\left(c_{1} c_{2} \gamma\right)^{n} x^{n(\gamma-1)} \cdot \exp \left(n c_{2} x^{\gamma}\right) \cdot \exp \left[c_{1} \exp \left(c_{2} x^{\gamma}\right)\right] ; n \geq 1 ; c_{i}, \gamma \neq 0 ; \\
& D^{n}\left((\log x)^{\delta}\right) \sim(-1)^{n-1}(n-1) ! \delta x^{-n}(\log x)^{\delta-1}, x \rightarrow+\infty ; n \geq 1 ; \delta \neq 0,1 ; \\
& D^{n}\left(\ell_{k}(x)\right) \sim(-1)^{n-1}(n-1) ! x^{-n}\left(\prod_{i=1}^{k-1} \ell_{i}(x)\right)^{-1}, x \rightarrow+\infty ; n \geq 1 ; k \geq 2 ; \\
& D^{n}\left(x^{\alpha}(\log x)^{\delta}\right) \sim \alpha^{\underline{n}} x^{\alpha-n}(\log x)^{\delta}, x \rightarrow+\infty ; n \geq 1 ; \alpha \in \mathbb{R} \backslash \mathbb{N} ; \delta \neq 0 .
\end{aligned}
$$

\section{Asymptotic Functional Equations for Regular or Rapid Variation}

Using representations in (2.12) it is easy to prove certain useful asymptotic relations satisfied by regularly-varying functions and in particular (2.6) which has been assumed by Karamata as the definition of a general concept of regular variation. The standpoint in this section is that of highlighting how a given function acts upon various asymptotic relations and we give these properties the collective name of "asymptotic functional equations".

Proposition 5.1. (Slow and regular variation). (I) A function $f \in \mathcal{R}_{0}(+\infty)$ enjoys the following asymptotic property.

$$
\left\{\begin{array}{ll}
g_{1}(x) \asymp g_{2}(x), & x \rightarrow+\infty, \\
g_{i}(x) \rightarrow+\infty, & x \rightarrow+\infty,(i=1,2)
\end{array} \Rightarrow f\left(g_{1}(x)\right) \sim f\left(g_{2}(x)\right), x \rightarrow+\infty ;\right.
$$

which states that a slowly-varying function transforms the relation of "asymptotic similarity between functions diverging to $+\infty$ ", see (1.11), into the stronger relation of "asymptotic equivalence": A property elementarily checked for the iterated logarithms and their powers. In particular $f$ satisfies the asymptotic functional equation:

$$
f(\lambda g(x)+o(g(x))) \sim f(g(x)), x \rightarrow+\infty,\left\{\begin{array}{l}
\text { for each fixed } \lambda>0 \text { and } \\
\text { for each g such that } g(+\infty)=+\infty,
\end{array}\right.
$$


which, by the presence of the parameter $\lambda$, says a bit more than "preserving asymptotic equivalence".

(II) A function $f \in \mathcal{R}_{\alpha}(+\infty), \alpha \in \mathbb{R}$, enjoys the following asymptotic properties:

$$
\begin{gathered}
\left\{\begin{array}{l}
g_{1}(x) \asymp g_{2}(x), x \rightarrow+\infty, \\
g_{i}(x) \rightarrow+\infty, x \rightarrow+\infty,(i=1,2)
\end{array} \Rightarrow f\left(g_{1}(x)\right) \asymp f\left(g_{2}(x)\right), x \rightarrow+\infty ;\right. \\
\left\{\begin{array}{l}
g_{1}(x) \sim \lambda g_{2}(x), x \rightarrow+\infty,(\lambda>0) \\
g_{i}(x) \rightarrow+\infty, x \rightarrow+\infty,(i=1,2)
\end{array} \Rightarrow f\left(g_{1}(x)\right) \sim \lambda^{\alpha} f\left(g_{2}(x)\right), x \rightarrow+\infty .\right.
\end{gathered}
$$

They mean that a regularly-varying function preserves the relations of "asymptotic similarity" and "asymptotic equivalence" between functions diverging to $+\infty$ with an addditional property concerning the multiplication of the argument by a constant factor $\lambda$. The last inference may be symbolically written as the asymptotic functional equation:

$$
f(\lambda g(x)+o(g(x))) \sim \lambda^{\alpha} f(g(x)), x \rightarrow+\infty,\left\{\begin{array}{l}
\text { for each fixed } \lambda>0 \text { and } \\
\text { for each g such that } g(+\infty)=+\infty
\end{array}\right.
$$

in particular.

$$
f(\lambda x+o(x)) \sim \lambda^{\alpha} f(x), x \rightarrow+\infty, \text { for each fixed } \lambda>0 .
$$

The asymptotic functional equation (2.6) is a special case of (5.6) and it expresses the "power-like" type of growth of $f$. For $\lambda=1$ we have the more precise result.

$r(x)=o(x), x \rightarrow+\infty, \Rightarrow f(x+r(x))=f(x)+x^{-1} r(x) f(x)[\alpha+o(1)], x \rightarrow+\infty$;

referring to $\$ 11$ in Part II for expansions with more terms. Two special cases of (5.7) are:

$$
\begin{gathered}
f(x+O(1))=f(x)+O\left(x^{-1} f(x)\right), x \rightarrow+\infty ; \\
f(x+\lambda+o(1))=f(x)+\lambda x^{-1} f(x)[\alpha+o(1)], x \rightarrow+\infty, \text { for each fixed } \lambda \neq 0 .
\end{gathered}
$$

Another useful consequence of (5.6) is.

$$
\int_{x}^{x+r(x)} f(t) \mathrm{d} t \sim r(x) f(x), x \rightarrow+\infty, \text { for } r(x)=o(x) ;
$$

and in particular.

$$
\int_{x}^{x+\lambda+o(1)} f(t) \mathrm{d} t \sim \lambda f(x), x \rightarrow+\infty, \text { for each fixed } \lambda \neq 0 .
$$

Proof. Rewrite (2.12) as

$$
f(x)=c \cdot \exp \left(\int_{T}^{x} \frac{f^{\prime}(t)}{f(t)} \mathrm{d} t\right) \text { with } c>0
$$

and $f^{\prime}(x) / f(x)=\alpha x^{-1}+o\left(x^{-1}\right), x \rightarrow+\infty$.

To prove (5.1) and (5.3) let $I, g$ be two functions defined on a neighborhood of $+\infty$ such that:

$$
0<c_{1} \leq I(x) \leq c_{2}<+\infty ; g(+\infty)=+\infty ;
$$


then

$$
\frac{f(I(x) g(x))}{f(g(x))}=\exp \left(\int_{g(x)}^{I(x) g(x)} \frac{f^{\prime}(t)}{f(t)} \mathrm{d} t\right)=\exp \left(\alpha \log I(x)+\int_{g(x)}^{I(x) g(x)} o\left(t^{-1}\right) \mathrm{d} t\right) .
$$

By (5.13):

$$
0<\bar{c}_{1} \leq|\log I(x)| \leq \bar{c}_{2}<+\infty
$$

and

$$
\forall \epsilon>0 \exists T_{\epsilon}: x>T_{\epsilon} \Rightarrow\left|\int_{g(x)}^{I(x) g(x)} o\left(t^{-1}\right) \mathrm{d} t\right|<\epsilon\left|\int_{g(x)}^{I(x) g(x)} t^{-1} \mathrm{~d} t\right|=\epsilon|\log I(x)|<\bar{C}_{2} \epsilon,
$$

so that

$$
\int_{g(x)}^{I(x) g(x)} o\left(t^{-1}\right) \mathrm{d} t=o(1), x \rightarrow+\infty .
$$

For $\alpha \neq 0$ we use these facts in the right-hand side of (5.14) so getting the inequalities

$$
0<d_{1} \leq \frac{f(I(x) g(x))}{f(g(x))} \leq d_{2}<+\infty \forall x \text { large enough, }
$$

which are the precise meaning of the thesis in (5.3). And for $\alpha=0$ the more precise relation in (5.1) is obtained. Analogously we have

$$
\begin{aligned}
\frac{f(\lambda g(x)+o(g(x)))}{f(g(x))} & =\exp \left(\int_{g(x)}^{\lambda g(x)+o(g(x))} \frac{f^{\prime}(t)}{f(t)} \mathrm{d} t\right) \\
& =\exp \left(\alpha \log (\lambda+o(1))+\int_{g(x)}^{\lambda g(x)+o(g(x))} o\left(t^{-1}\right) \mathrm{d} t\right) \\
& \stackrel{\text { by (5.17) }}{=} \exp (\alpha \log \lambda+o(1))=\lambda^{\alpha}+o(1),
\end{aligned}
$$

which is (5.4), equivalent to (5.5). The asymptotic expansion in (5.7) is similarly proved:

$$
\begin{aligned}
\frac{f(x+r(x))}{f(x)} & =\exp \left(\int_{x}^{x+r(x)} \frac{f^{\prime}(t)}{f(t)} \mathrm{d} t\right)=\exp \left[\alpha \log \left(1+\frac{r(x)}{x}\right)+o\left(\log \left(1+\frac{r(x)}{x}\right)\right)\right] \\
& =\exp \left[\alpha \frac{r(x)}{x}+o\left(\frac{r(x)}{x}\right)\right]=1+\alpha \frac{r(x)}{x}+o\left(\frac{r(x)}{x}\right) .
\end{aligned}
$$

Last, (5.10) follows from the mean-value theorem of integral calculus:

$$
\int_{x}^{x+r(x)} f(t) \mathrm{d} t=r(x) f(x+\theta(x) r(x)) \stackrel{\text { by }(5.5)}{\sim} r(x) f(x),
$$

as " $\theta(x) r(x)=o(x)$ ". An alternative proof, only valid for $\alpha \neq-1$, follows from (5.7) applied to an antiderivative of $f$, say $F$, which satisfies $F \in \mathcal{R}_{\alpha+1}(+\infty)$, by writing

$$
\int_{x}^{x+r(x)} f=F(x+r(x))-F(x) \sim(\alpha+1) x^{-1} F(x) r(x),
$$

and then using (2.78) or (2.79). $\square$

A comment on uniform convergence. Refining the calculations in (5.19) the last ex- 
pression in (5.19) may be replaced by

$$
\exp \left(\alpha \log \lambda+\alpha \log \left(1+\frac{o(1)}{\lambda}\right)+I(x)\right), I(x):=\int_{g(x)}^{\lambda g(x)+o(g(x))} o\left(t^{-1}\right) \mathrm{d} t,
$$

where the symbols " $O(\cdot)$ " stand for suitable functions not dependent on $\lambda$. Hence:

$$
\frac{f(\lambda g(x)+o(g(x)))}{\lambda^{\alpha} f(g(x))}-1=\left(1+\frac{o(1)}{\lambda}\right)^{\alpha} \cdot \exp I(x)-1
$$

and it is easily seen that the quantity on the right tends to zero, as $x \rightarrow+\infty$, uniformly with respect to $\lambda$ varying on any compact interval of type $\left[c_{1}, c_{2}\right], 0<c_{1}<c_{2}<+\infty$. This fact, elementary for regular variation in the strong sense, is a nontrivial result for regular variation in Karamata's sense, basic for the whole theory: Uniform Convergence Theorem ([8]; pp. 6-10 and pp. 22-23). A minor result in the general theory states that the asymptotic functional equation (2.6) is equivalent to the (seemingly more general) (5.6).

In the next proposition special cases of the asymptotic relation in (5.7) are commented upon.

Proposition 5.2. (I) (Asymptotic sublinearity). If $f \in \mathcal{R}_{\alpha}(+\infty), \alpha<1$, then (2.19) implies that $x^{-1} f(x)=o(1)$ and (5.8) becomes:

$$
f(x+O(1))=f(x)+o(1), x \rightarrow+\infty,
$$

a property enjoyed by all functions $f \in A C[T,+\infty)$ such that $f^{\prime}(x)=o(1), x \rightarrow+\infty$, as follows at once from representation

$$
f(x+r(x))-f(x)=\int_{x}^{x+r(x)} f^{\prime}(t) \mathrm{d} t
$$

Such a property may be interpreted as a kind of "asymptotic sublinearity".

(II) (Asymptotic linearity). For a function $f \in \mathcal{R}_{1}(+\infty)$ and

$f(x) \sim A x, x \rightarrow+\infty, A \neq 0$, the asymptotic functional equation in (5.9) becomes:

$$
f(x+\lambda+o(1))=f(x)+A \lambda+o(1), x \rightarrow+\infty \text {, for each fixed } \lambda \neq 0,
$$

a property enjoyed by all functions $f \in A C[T,+\infty)$ such that $f^{\prime}(x)=A+o(1), x \rightarrow+\infty$, as again follows from representation (5.26). An instance is provided by the function $\phi$ defined in (3.24) and associated to $f$ in defining the concept of smooth variation. (As we know, the sole condition $f(x) \sim A x, A \neq 0$, implies " $f$ regularly varying at $+\infty$ of index 1 in the general sense of Karamata".)

The property in (5.27) may be interpreted as a kind of "asymptotic linearity". For $\lambda=0$ (5.27) takes the form:

$$
f(x+o(1))=f(x)+o(1), x \rightarrow+\infty,
$$

an equation satisfied by all functions $f \in A C[T,+\infty)$ such that $f^{\prime}(x)=O(1), x \rightarrow+\infty$, a meaningful subclass being that of the functions ultimately "positive and concave". A proper label for the property in (5.28) is "asymptotic uniform continuity at $+\infty$ " as it can be proved that. 
" $A$ function $f:[T,+\infty) \rightarrow \mathbb{R}$ is uniformly continuous on $[T,+\infty)$ iff it is continuous on $[T,+\infty)$ and asymptotically uniformly continuous at $+\infty$ ".

(III) (A subclass of slowly-varying functions). The strong asymptotic functional equation

$$
f(x+o(x))=f(x)+o(1), x \rightarrow+\infty,
$$

states that $f$ transforms the relation of "asymptotic equivalence between functions diverging to $+\infty$ " into the stronger relation of "asymptotic equivalence with an infinitesimal remainder". Representation in (5.26) easily implies that (5.29) is satisfied by any $f \in A C[T,+\infty)$ such that $f^{\prime}(x)=O\left(x^{-1}\right)$, for instance by all functions " $f \in \mathcal{R}_{0}(+\infty)$ and bounded" and by.

$$
\left\{\begin{array}{l}
(\log x)^{\delta}, \delta \leq 1 ;\left(\ell_{k}(x)\right)^{\delta}, k \geq 2, \delta \in \mathbb{R} ; \\
\prod_{1 \leq k \leq n}\left(\ell_{k}(x)\right)^{\delta_{k}} \text {, where either }\left\{\delta_{1}<1, \delta_{k} \in \mathbb{R}\right\} \text { or }\left\{\delta_{1}=1, \delta_{k} \leq 0\right\} ;
\end{array}\right.
$$

but neither by $(\log x)^{\delta}, \delta>1$, nor by $\exp \left[(\log x)^{\delta}\right], \delta>0$.

For rapid variation, which formally refers to the limit cases $\alpha= \pm \infty$ of regular variation, only a formal analogue of (5.5) holds true.

Proposition 5.3. (Rapid variation). (I) If $f \in \mathcal{R}_{+\infty}(+\infty)$ and if $\lim _{x \rightarrow+\infty} g(x)=+\infty$ then:

$$
\lim _{x \rightarrow+\infty} \frac{f(\lambda g(x)+o(g(x)))}{f(g(x))}= \begin{cases}0 & \text { if } 0<\lambda<1 \\ +\infty & \text { if } 1<\lambda<+\infty\end{cases}
$$

In particular

$$
\lim _{x \rightarrow+\infty} f(\lambda x) / f(x)= \begin{cases}0 & \text { if } 0<\lambda<1, \\ +\infty & \text { if } 1<\lambda<+\infty .\end{cases}
$$

(II) If $f \in \mathcal{R}_{-\infty}(+\infty)$ and if $\lim _{x \rightarrow+\infty} g(x)=+\infty$ then:

$$
\lim _{x \rightarrow+\infty} \frac{f(\lambda g(x)+o(g(x)))}{f(g(x))}= \begin{cases}+\infty & \text { if } 0<\lambda<1, \\ 0 & \text { if } 1<\lambda<+\infty .\end{cases}
$$

In particular

$$
\lim _{x \rightarrow+\infty} f(\lambda x) / f(x)= \begin{cases}+\infty & \text { if } 0<\lambda<1, \\ 0 & \text { if } 1<\lambda<+\infty .\end{cases}
$$

The asymptotic functional relations (5.32) and (5.34) where assumed by de Haan as definitions of the general classes of (measurable) rapidly-varying functions of index $\pm \infty$, respectively: ([8]; p. 83).

Proof. (I) From relation (2.2) we get that $\forall M>0 \exists T_{M}$ such that $x>T_{M}$ implies " $f^{\prime}(x) / f(x) \geq M$ ", and for all $x$ large enough we also have:

$$
\begin{gathered}
\int_{g(x)}^{\lambda g(x)+o(g(x))} \frac{f^{\prime}(t)}{f(t)} \mathrm{d} t \geq M \int_{g(x)}^{\lambda g(x)+o(g(x))} t^{-1} \mathrm{~d} t=M \log (\lambda+o(1)), \text { if } \lambda>1, \\
-\int_{\lambda g(x)+o(g(x))}^{g(x)} \frac{f^{\prime}(t)}{f(t)} \mathrm{d} t \leq-M \log \left(\frac{g(x)}{\lambda g(x)+o(g(x))}\right)=M \log (\lambda+o(1)), \text { if } 0<\lambda<1,
\end{gathered}
$$


whence

$$
\lim _{x \rightarrow+\infty} \int_{g(x)}^{\lambda g(x)+o(g(x))} \frac{f^{\prime}(t)}{f(t)} \mathrm{d} t= \begin{cases}-\infty & \text { if } 0<\lambda<1 \\ +\infty & \text { if } 1<\lambda<+\infty .\end{cases}
$$

The limits in (5.31) follow by applying the exponential as in (5.19) and those in (5.33) follow by applying the just-proved result to the function $1 / f$

\section{References}

[1] Granata, A. (2011) Analytic Theory of Finite Asymptotic Expansions in the Real Domain. Part I: Two-Term Expansions of Differentiable Functions. Analysis Mathematica, 37, 245-287. http://dx.doi.org/10.1007/s10476-011-0402-7

[2] Granata, A. (2015) The Factorizational Theory of Finite Asymptotic Expansions in the Real Domain: A Survey of the Main Results. Advances in Pure Mathematics, 5, 1-20. http://dx.doi.org/10.4236/apm.2015.51001

[3] Granata, A. (2015) Analytic Theory of Finite Asymptotic Expansions in the Real Domain. Part II-A: The Factorizational Theory for Chebyshev Asymptotic Scales. Advances in Pure Mathematics, 5, 454-480. http://dx.doi.org/10.4236/apm.2015.58045

[4] Granata, A. (2015) Analytic Theory of Finite Asymptotic Expansions in the Real Domain. Part II-B: Solutions of Differential Inequalities and Asymptotic Admissibility of Standard Derivatives. Advances in Pure Mathematics, 5, 481-502. http://dx.doi.org/10.4236/apm.2015.58046

[5] Granata, A. (2015) Analytic Theory of Finite Asymptotic Expansions in the Real Domain. Part II-C: Constructive Algorithms for Canonical Factorizations and a Special Class of Asymptotic Scales. Advances in Pure Mathematics, 5, 503-526. http://dx.doi.org/10.4236/apm.2015.58047

[6] Bourbaki, N. (1976) Fonctions d’une Variable Réelle-Théorie Élémentaire. Hermann, Paris.

[7] Balkema, A.A., Geluk, J.L. and de Haan, L. (1979) An Extension of Karamata's Tauberian Theorem and Its Connection with Complementary Convex Functions. The Quarterly Journal of Mathematics, Oxford University Press, Series 2, 30, 385-416. http://dx.doi.org/10.1093/qmath/30.4.385

[8] Bingham, N.H., Goldie, C.M. and Teugels, J.L. (1987) Regular Variation. Cambridge University Press, Cambridge. http://dx.doi.org/10.1017/CBO9780511721434

[9] Lantsman, M.H. (2001) Asymptotics of Linear Differential Equations. Springer Science+ Business Media, Dordrecht. http://dx.doi.org/10.1007/978-94-015-9797-5

[10] Seneta, E. (1976) Regularly Varying Functions. Springer-Verlag, Berlin-Heidelberg-New York. http://dx.doi.org/10.1007/BFb0079658

[11] Pólya, G. and Szëgo, G. (1972) Problems and Theorems in Analysis. Vol. I. Springer-Verlag, Berlin-Heidelberg-New York. http://dx.doi.org/10.1007/978-1-4757-1640-5

[12] Granata, A. (2010) The Problem of Differentiating an Asymptotic Expansion in Real Powers. Part I: Unsatisfactory or Partial Results by Classical Approaches. Analysis Mathematica, 36, 85-112. http://dx.doi.org/10.1007/s10476-010-0201-6

[13] Granata, A. (2015) The Role of Asymptotic Mean in the Geometric Theory of Asymptotic Expansions in the Real Domain. Advances in Pure Mathematics, 5, 100-119. http://dx.doi.org/10.4236/apm.2015.52013 
Submit or recommend next manuscript to SCIRP and we will provide best service for you:

Accepting pre-submission inquiries through Email, Facebook, LinkedIn, Twitter, etc. A wide selection of journals (inclusive of 9 subjects, more than 200 journals)

Providing 24-hour high-quality service

User-friendly online submission system

Fair and swift peer-review system

Efficient typesetting and proofreading procedure

Display of the result of downloads and visits, as well as the number of cited articles

Maximum dissemination of your research work

Submit your manuscript at: http://papersubmission.scirp.org/

Or contact apm@scirp.org 